\title{
Improved parameterization of phosphatidylinositide lipid headgroups for the Martini 3 coarse grain force field
}

\author{
L. Borges-Araújo $+\|, \perp$, P. C. T. Souza $\S$, F. Fernandes $\uparrow, \neq, \perp$, M. N. Melo $\|^{\|, *}$ \\ † iBB-Institute for Bioengineering and Biosciences, Instituto Superior Técnico, Universidade de Lisboa, Lisbon, \\ Portugal
}

$\perp$ Associate Laboratory i4HB-Institute for Health and Bioeconomy at Instituto Superior Técnico, Universidade de Lisboa, Lisbon, Portugal

‡ Department of Bioengineering; Instituto Superior Técnico, Universidade de Lisboa, 1049-001 Lisbon, Portugal;

$\S$ Molecular Microbiology and Structural Biochemistry, UMR 5086 CNRS \& University of Lyon, 7 Passage du Vercors, F-69367, Lyon, France.

|| Instituto de Tecnologia Química e Biológica António Xavier, Universidade Nova de Lisboa, Av. da República, 2780157 Oeiras, Portugal.

\begin{abstract}
Phosphoinositides are a family of membrane phospholipids that play crucial roles in membrane regulatory events. As such, these lipids are often a key part of molecular dynamics simulation studies of biological membranes, in particular of those employing coarse-grain models because of the potential long times and sizes of the involved membrane processes. Version 3 of the widely used Martini coarse grain force field has been recently published, greatly refining many aspects of biomolecular interactions. In order to properly use it for lipid membrane simulations with phosphoinositides, we put forth the Martini 3-specific parameterization of inositol, phosphatidylinositol, the seven physiologically relevant phosphorylated derivatives of phosphatidylinositol. Compared to parameterizations for earlier Martini versions, focus was put on a more accurate reproduction of the behavior seen in both atomistic simulations and experimental studies, including the signalingrelevant phosphoinositide interaction with divalent cations. The models we develop improve upon the conformational dynamics of phosphoinositides in the Martini force field and provide stable topologies at typical Martini timesteps. They are able to reproduce experimentally known protein-binding poses as well as phosphoinositide aggregation tendencies. The latter were tested both in the presence and absence of calcium, and include correct behavior of $\mathrm{PI}(4,5) \mathrm{P}_{2}$ calcium-induced clusters, which can be of relevance for regulation.
\end{abstract}

\section{INTRODUCTION}

Phosphoinositides are a small group of glycerophospholipids, derived from the reversible phosphorylation of phosphatidylinositol (PI), that account for around 10 to $15 \%$ of the total membrane phospholipid content of eukaryotic cells ${ }^{1}$. While these lipids are minor components of eukaryotic biomembranes, research over the last couple of decades has established their role as major regulators of cell dynamics and signaling in eukaryotic metabolism. Their impact extends far beyond the cell membrane, where they take part in regulating several processes such as endocytosis/exocytosis ${ }^{1-5}$, ion channel regulation ${ }^{6-8}$, cellular signaling ${ }^{1,2}$ or cytoskeleton reorganization ${ }^{1,2,9}$, impacting downstream processes such as cellular proliferation, migration, survival and differentiation ${ }^{10}$. Additionally, as it often happens with ubiquitous signaling entities, disorders affecting their metabolism are responsible for several human conditions ${ }^{7}$ which can range from neurological pathologies ${ }^{7,11}$ (such as Charcot-Marie-Tooth neuropathy) to cancer ${ }^{12,13}$.

The core structure of every phosphoinositide lipid is inherited from PI. PI is composed of a diacylglycerol con- nected to a distinctive myo-inositol ring by a phosphodiester bond. While there are 9 possible isomers of inositol, myo-inositol is the isomer most commonly found in eukaryotic cells ${ }^{14}$. It assumes a chair conformation where every hydroxyl substituent is at the equatorial position, except for the hydroxyl group in the position 2 of the ring (Figure 1). In forming PI, the hydroxyl group in position 1 of myo-inositol takes part in the phosphodiester bond with the diacylglycerol backbone. The hydroxyl groups in positions 3,4 , and 5 of the PI ring can be enzymatically phosphorylated in every possible combination, yielding the seven phosphorylated phosphoinositide species found in mammalian cells (Figure 1): phosphatidylinositol 3-phosphate (PI(3)P), phosphatidylinositol 4-phosphate (PI(4)P), phosphatidylinositol 5-phosphate (PI(5)P), phosphatidylinositol 3,4-bisphosphate $\left(\mathrm{PI}(3,4) \mathrm{P}_{2}\right)$, phosphatidylinositol 3,5bisphosphate $\left(\mathrm{PI}(3,5) \mathrm{P}_{2}\right)$, phosphatidylinositol 4,5-bisphosphate $(\mathrm{PI}(4,5) \mathrm{P} 2)$ and phosphatidylinositol 3,4,5-trisphosphate $\left(\mathrm{PI}(3,4,5) \mathrm{P}_{3}\right)$.

While PI is synthesized in the endoplasmic reticulum and then distributed throughout the cell, each of the phosphorylated species has a distinct subcellular distribution within 


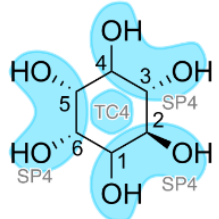

myo-Inositol
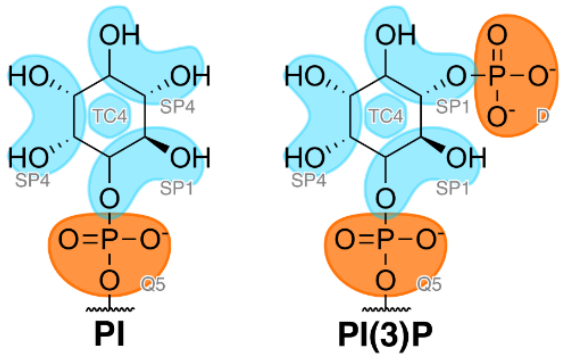
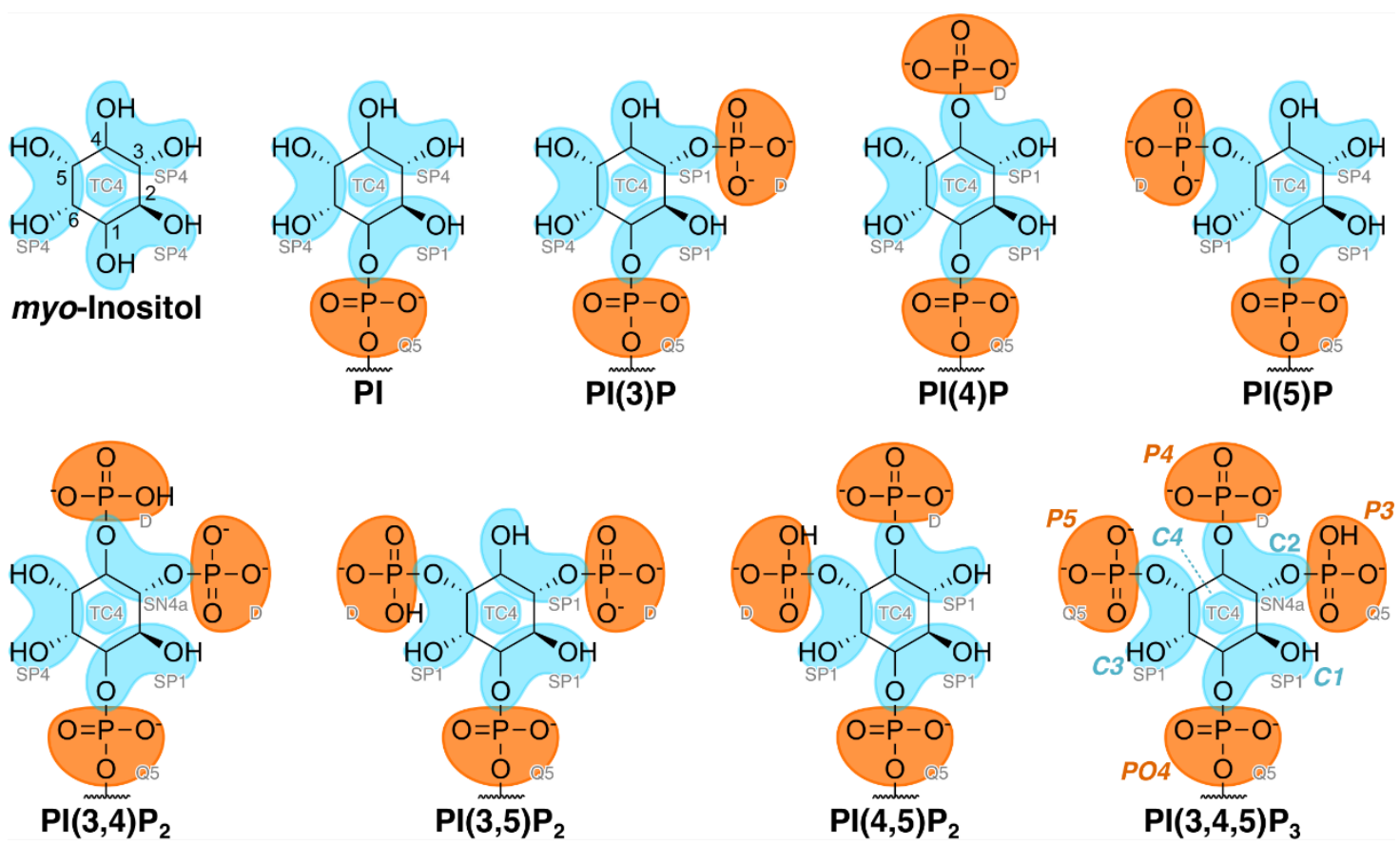

Figure 1. Chemical structures and coarse-grained mapping of the inositol/phosphoinositides parameterized in this work. The inositol ring carbon numbering is overlaid on the myo-inositol structure. Atom-to-bead mappings are indicated by the colored shapes, with an extra bead assigned to the center of the inositol ring (ring beads in blue, phosphate beads in orange). Assigned Martini 3 bead types are indicated for each bead as overlaid gray text. In the structure of $\mathrm{PI}(3,4,5) \mathrm{P}_{3}$, in which all possible beads are present, bead names are indicated as bold-italic colored text (following the convention in this text that bead names are typed in italics, to distinguish from bead types).

subsets of membranes as a result of the localization of specific PI-kinases and phosphatases that catalyze their local formation from PI. Apart from their distinct distributions, each phosphoinositide species also establishes distinct interactions with specific binding partners. Phosphoinositides achieve most of their direct signaling effects through interactions between their headgroup and protein partners. While many of these are transient, low-affinity interactions with basic residues within unstructured protein domains, high-affinity, specific interactions with specialized domains also occur, some of which are able to distinguish between phosphoinositide isomers ${ }^{2}$. Their distinct subcellular localization, combined with the distinct set of interactions established with cellular binding partners, grants each isomer specific roles and regulatory functions within the cell.

Additionally, some phosphoinositides and $\mathrm{PI}(4,5) \mathrm{P}_{2}$, in particular, are able to establish strong electrostatic interactions with divalent cations $\left(\mathrm{Ca}^{2+}\right.$ and $\left.\mathrm{Mg}^{2+}\right)$, culminating in the cation-dependent aggregation into stable phosphoinositide nanodomains ${ }^{15-17}$. These are formed at physiological concentrations of both lipid and divalent cations, and it is likely that some are found constitutively clustered in vivo. While these nanodomains are often overlooked, they potentially impact phosphoinositide-protein interactions.

The wide-range influence of the phosphoinositide family has piqued research interest, leading to numerous of experimental and theoretical research studies ${ }^{7}$. Theoretical studies, such as molecular dynamics (MD) simulations have the potential to provide molecular level structural and dynamic insight on complex systems, such as biological membranes, that is otherwise inaccessible through experiments. This gap in knowledge is particularly evident in the case of phosphoinositides, where there is still a considerable lack of understanding about many of the molecular mechanisms behind their function, such as lateral organization, interactions with binding partners, cation-induced nanodomain formation or induction of curvature.

While MD studies at an all-atom (AA) level of resolution of lipid membrane systems can be performed, they are still limited by current computational power in terms of attainable system size and time scales. Coarse-grained (CG) MD models, however, have allowed access to scales of tens-tohundreds of $\mathrm{nm}$, and into the millisecond - termed the mesoscale - at which many of the phosphoinositide processes become of relevance ${ }^{18}$. The Martini CG model ${ }^{19}$ is the most widely used CG force field for biomolecular simulations ${ }^{20,21}$ and it has been successfully applied in the modeling of a variety of molecular processes - especially, in the modeling of biomembrane systems. The large-scale Martini simulation of an average mammalian plasma membrane model ${ }^{18}$ stands out as one of the most complex simulation to date. Neuronal plasma membrane systems, where phosphoinositides are thought to play an important role, have also been simulated ${ }^{22}$. Martini applications extend to phosphoinositide-protein interactions, having been able to predict the location of PI $(4,5) \mathrm{P}_{2}$ binding sites on Kir channels ${ }^{23}$, later confirmed by $\mathrm{PI}(4,5) \mathrm{P}_{2}$-bound protein crystal structures ${ }^{24}$.

Martini is based on a four-to-one mapping scheme, meaning that on average, four heavy (non-hydrogen) atoms, and 
A
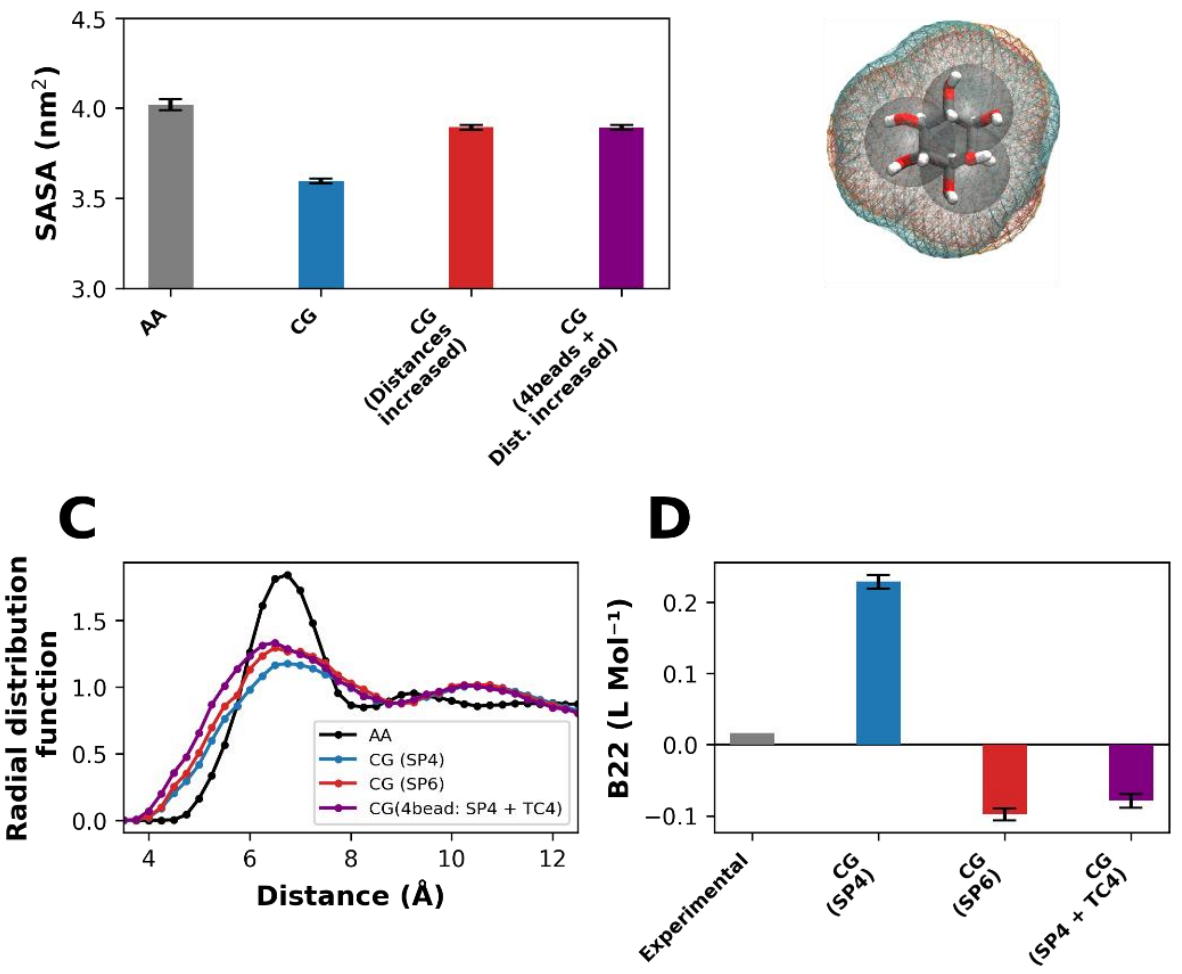

B
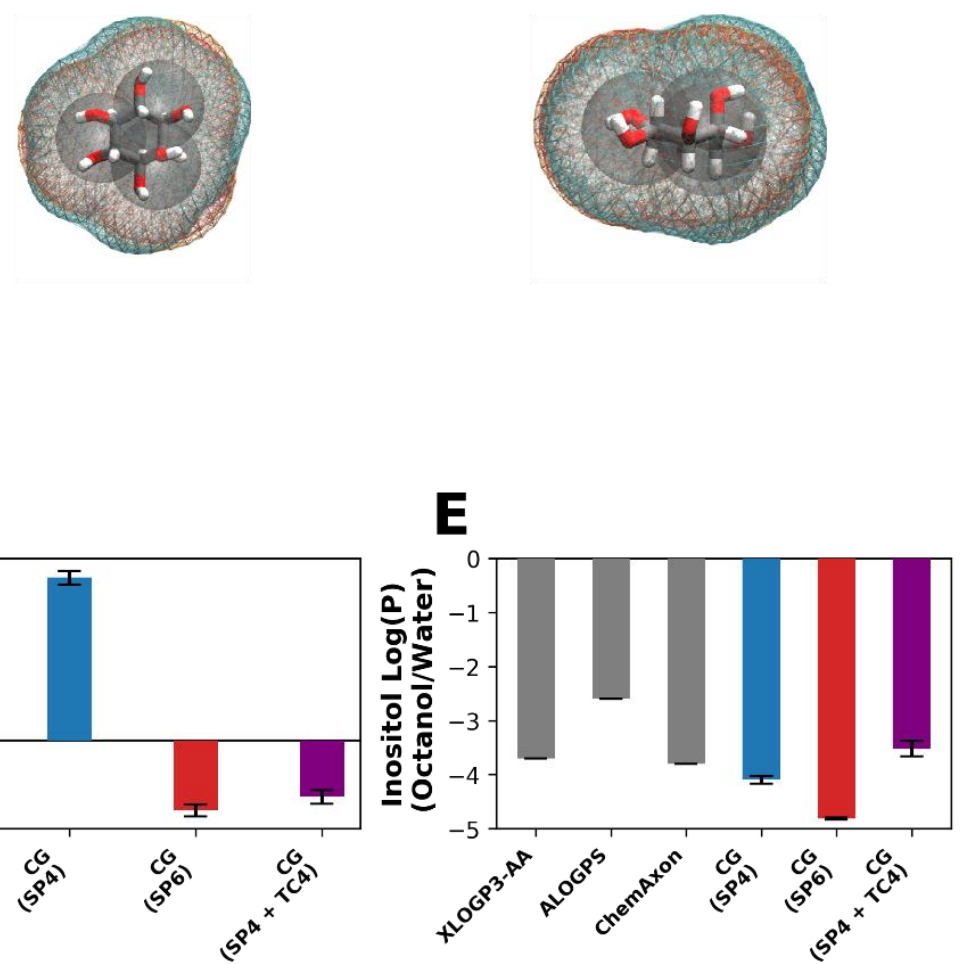

Figure 2. myo-Inositol Martini 3 CG model properties. SASA (A), inositol c.o.m. vs inositol c.o.m. RDF (C), B22 osmotic coefficient (D), and inositol $\log (\mathrm{P})$ values (E) for several iterations of the inositol CG model as well as the appropriate references. Error bars for the B22 measurements (D) represent the standard error of the mean determined from 16200 ns measurements over the course of $3.5 \mu$ s (Figure S1). Otherwise, when applicable, error bars represent the standard deviation from 3 independent simulation experiments. Connolly surfaces (B) for 2 iterations of the CG inositol model (c.o.g.-mapped bead distance: red wireframe; $20 \%$ increased bead distance: orange wireframe) as well as the atomistic reference (blue wireframe).

associated hydrogens, are represented as a single CG particle, or 'bead'. Each bead is assigned a type dependent on its underlying chemical nature in terms of polarity and charge. Different interparticle non-bonded potentials are associated to the interactions between each type, effectively encoding a bead's interaction preferences. Martini molecules are then assembled from beads in a building-block fashion, employing 2-, 3- and 4-body bonded potentials (for bonds, angles, and torsions, respectively) to define preferred configurations - matching known behavior from either experiments or from finer simulation sources - and delimiting the overall explorable molecular configuration space.

Within the Martini philosophy, bead type nonbonded potentials have been parameterized to reproduce thermodynamic properties of the represented chemical moieties - in particular, the partitioning between polar and nonpolar phases. It is an underlying assumption that, when bonded together, the additive properties of the individual Martini beads can overall represent the properties of the entire modeled molecule. However, in Martini 2 this was found to not hold in specific cases where mappings finer than 4-to-1 were employed, or when intermolecular bead-bead distances were very short. This led to a number of non-obvious pitfalls ${ }^{20}$, the most important being the observations that some molecules become too hydrophobic or self-interact too strongly - in particular proteins ${ }^{20,25-27}$ and carbohydrates $^{20,28}$, such as phosphoinositides. Indeed, phospho- inositides included in the pioneering Martini plasma membrane study were found to form dimers and trimers more frequently than expected ${ }^{18}$.

A new version of the Martini force field, Martini 3, has been recently released ${ }^{29}$. This new implementation of the force field addresses the major limitations of Martini 2 by including bead types specific for mappings finer than 4-to1 . The release of the new force field version also greatly increases the discrimination of chemical space by defining a larger number of bead types, allowing for more flexibility and accuracy when creating new models. Besides expanding the nonbonded landscape, the Martini 3 release also establishes new guidelines for the bonded parameterization of molecules in tandem with the new bead types. In this sense, the new Martini release provides a good opportunity to revisit, update and improve existing models.

The Martini 2 parameters for phosphatidylinositol and two of its phosphorylated forms were first parameterized by Lopez et al. ${ }^{30}$ using the GROMOS53A6 atomistic force field as reference. In this initial effort, headgroup motion was left mostly unrestrained, with only one torsion potential controlling it - and even this potential ended up being routinely ignored in production simulations ${ }^{31}$ as it led to numerical instability at typical Martini timesteps ${ }^{32}$. Different improvements on these Martini 2 parameters have been proposed $^{18,22}$, mostly focused on stabilizing bonded behav- 
ior. These approaches are still limited by the Martini 2 nonbonded insufficiencies and by an overall excessive freedom of the inositol ring.

In this work, we parameterize inositol and the 8 phosphoinositide lipids for Martini 3, using the CHARMM36 atomistic force field as structural atomistic reference. Besides compatibility with the rest of Martini 3 landscape, Martini 3 phosphoinositide models are expected to have more accurate interaction propensities than their Martini 2 counterparts. This parameterization effort is also an opportunity to expand the covered species to include all relevant phosphorylations. Finally, a new take on the parameterization of phosphoinositides can aim at better modeling i) their complex structural dynamics ${ }^{33}$ - a result of large headgroups, networks of intra-/inter-molecular hydrogen bonds and variable phosphate protonations - and ii) the often overlooked interaction of phosphoinositides with divalent cations, which can drive nanodomain formation of physiological relevance - especially involving the $\mathrm{PI}(4,5) \mathrm{P}_{2}$ headgroup $^{15,17}$.

\section{METHODS}

All simulations were run using the GROMACS simulation package version $2019^{34}$ and analyzed making use of inhouse developed Python3 programs using the MDAnalysis package $^{35}$. We also used the IPython ${ }^{36}$, numpy ${ }^{37}, \mathrm{SciPy}^{38}$, scikit-learn ${ }^{39}$, Voro ++40 and matplotlib ${ }^{41}$ packages for scientific computing in Python. Visualization and rendering of the simulations was performed with the molecular graphics viewer $\mathrm{VMD}^{42}$. Octanol-water partition free-energies were calculated from the individual CG solvation free-energies into each solvent, as described elsewhere ${ }^{43}$. See the Methods section of the Supporting Information for details on specific analysis methods.

Atomistic models. All atomistic models used as the parameterization targets were simulated using the CHARMM36 ${ }^{44,45}$ force field, with the TIP3P water model. All topologies used are readily available in CHARMM-GUI ${ }^{46,47}$. The topologies used for each of the parameterization targets can be seen in SI Material, table S1.

To validate the parameterization of the inositol molecule, simulations were performed of myo-inositol in water, either singly or as a solution of eight molecules. For single myoinositol molecule systems, the sugar molecule was inserted in a $5 \times 5 \times 5 \mathrm{~nm}$ simulation box, which was then fully solvated. As for the multiple myo-inositol system, eight sugars were placed in a cubic box and solvated at an 8.0 water/sugar (weight/weight) concentration.

For the lipid membrane systems initial structures were all generated, using the CHARMM-GUI membrane builder module ${ }^{48-51}$, by arranging the lipids on a regular array in the bilayer (xy) plane. Membranes were built with roughly 240 lipids per leaflet, which were then solvated by 11000 water molecules. Every system was neutralized and an additional $140 \mathrm{mM} \mathrm{NaCl}$ was added to better represent physiological conditions.

After initial energy minimization and equilibration runs, all atomistic simulations were run at a 2 fs time step. The LINCS $^{52}$ algorithm was applied to constrain all bonded hydrogens. Van der Waals forces were switched off smoothly from 1.0 to $1.2 \mathrm{~nm}$, and electrostatics were computed using particle-mesh Ewald summation ${ }^{53}$. The particle neighbor list was updated using the Verlet list scheme. System temperature was maintained at $310 \mathrm{~K}$ by coupling to a NoséHoover thermostat ${ }^{54}$ with a 1 ps coupling constant, while pressure was coupled to 1.0 bar using a Parrinello-Rahman barostat $^{55}$ (isotropically for aqueous systems, semi-isotropically for membrane systems) with a 5 ps coupling time.

All atomistic simulations were run for at least $2 \mu$ s and, unless otherwise stated, the last $1 \mu$ s was used for analysis.

Coarse-grained models. All coarse-grained simulations were modelled using the Martini 3 CG model for biomolecular simulations ${ }^{29}$. All topologies, other than the ones parameterized here, are available alongside the force field ${ }^{29}$. Along this text, bead names will be typed in italics, to distinguish them from bead types.

To validate the inositol headgroup, CG simulations were performed of a single myo-inositol sugar molecule in water as well as of multiple sugars, as done with the atomistic models.

For the lipid membrane systems initial structures were built and solvated using the insane.py CG building tool ${ }^{56}$ by arranging the lipids on a regular array in the bilayer $(x y)$ plane to obtain roughly 180 lipids per leaflet, solvated by roughly 8000 water beads. Larger systems containing up to 700 lipids per leaflet were also built for characterization of inositide lipid properties and $\mathrm{Ca}^{2+}$-induced inositide clustering. Counterions were added to neutralize the systems as necessary, plus $140 \mathrm{mM} \mathrm{NaCl}$ ionic strength. When testing divalent cation aggregation, $\mathrm{Ca}^{2+}$ was added to the systems at a 5:1 $\mathrm{Ca}^{2+}$-to-phosphoinositide ratio, by replacing water particles and maintaining the system charge neutral by adding $\mathrm{Cl}^{-}$counterions.

Two different proteins were also studied. Protein structures were obtained from the Protein Data Bank ${ }^{57}$ (PDB) (PLC81 PH domain PDB: $1 \mathrm{MAI}^{58}$, and the Kir2.2 channel PDB: 6M8459). All CG protein models were constructed using Martinize $2^{60}$, with an applied elastic network with a bond force constant of $700 \mathrm{~kJ} / \mathrm{mol}$ and a cut off distance of $0.8 \mathrm{~nm}$. Side chain corrections were also applied ${ }^{61}$. Both proteins were placed in lipid membrane systems. The CG model of the $\mathrm{PH}$ domain was placed in the corner of the simulation box with a random initial orientation. The CG model of the Kir2.2 channel was positioned roughly as described in previous simulation studies ${ }^{23,62}$.

Nonbonded interactions were cutoff at $1.1 \mathrm{~nm}$ and Coulombic interactions were treated using reaction-field electrostatics ${ }^{63}$ with a dielectric constant of 15 and an infinite reaction-field dielectric constant. The particle neighbor list was updated using the Verlet list scheme. The v-rescale thermostat $^{64}$ was used with a coupling time of 4.0 ps to maintain the temperature at $310 \mathrm{~K}$ (unless other temperature is specified). Constant pressure was isotropically or semi-isotropically (in the case of membranes) coupled to 1.0 bar using the Parrinello-Rahman barostat ${ }^{55}$ with a relaxation time of $16.0 \mathrm{ps}$. After initial energy minimization and pressure/temperature equilibration runs, simulations were run at a 20 fs time step. 

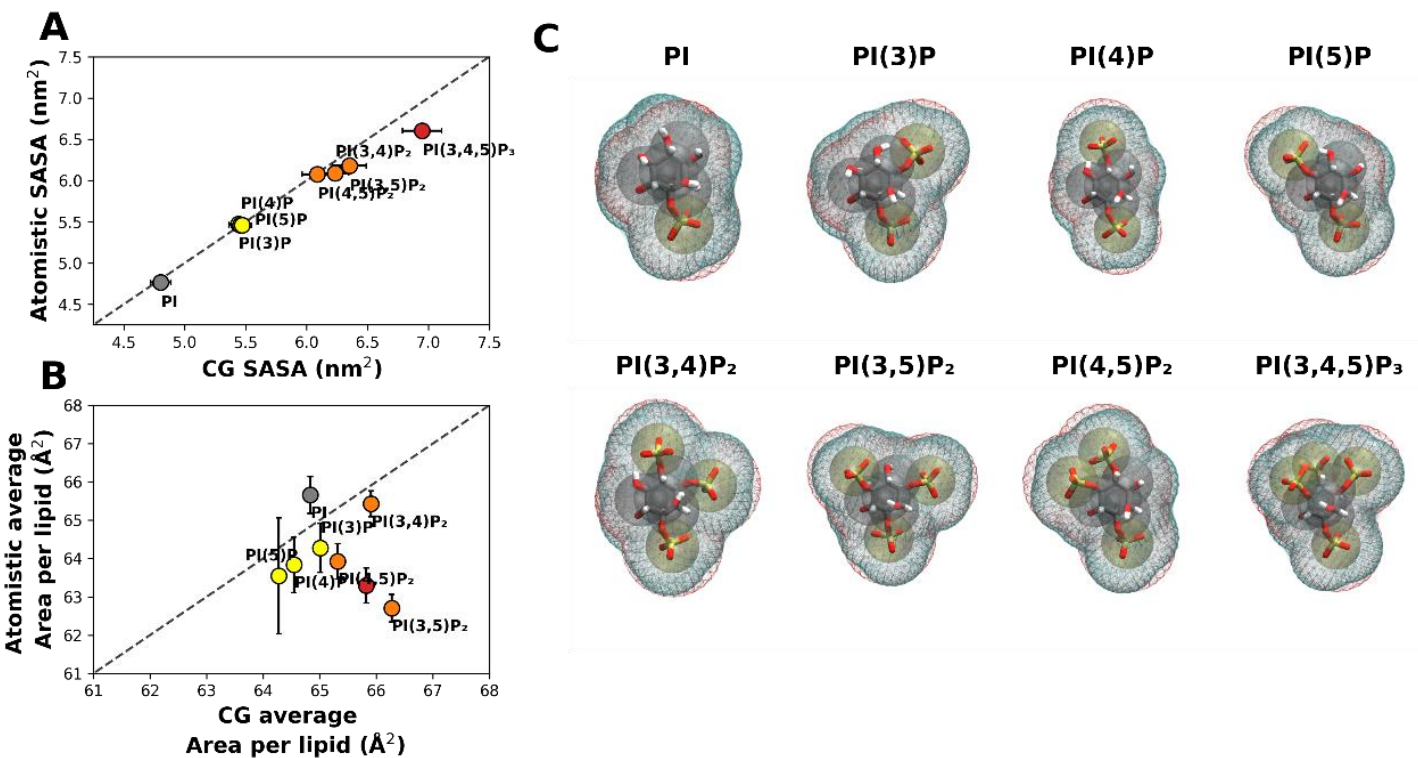

Area per lipid $\left(\AA^{2}\right)$

Figure 3. Phosphoinositide lipid headgroup SASA, Connolly surfaces and APL. Comparison of phosphoinositide headgroup SASA values (A) and APL values (B) recovered for the martini 3.0 and AA models. Colors indicate lipid phosphorylation (grey: No headgroup phosphates, yellow: mono-phosphorylated, orange: bis-phosphorylated and red: tris-phosphorylated). The $y=x$ relation was drawn as a guide. Connolly surfaces (C) were determined from AA and CG mapped structures which were then overlayed on top of each other. The AA surface is represented in blue and the CG surface in red.

All CG systems were run for at least $10 \mu$ s and unless otherwise stated the last $2 \mu$ s of each simulation were used for subsequent analysis.

All developed models are provided as GROMACScompatible topology files, as Supporting Information associated to this manuscript. Current and future parameter iterations can be tracked in the associated GitHub repository at https://github.com/MeloLab/PhosphoinositideParameters.

\section{Results and Discussion}

Our aim was to develop phosphoinositides parameters up to date with the new Martini 3 framework, also taking into account important phosphoinositide biophysical properties not previously accounted for - such as protonation and interaction with divalent cations. An overview of the mappings used in this work is given in Figure 1. While previous work on Martini 2 phosphoinositides served as inspiration for some of our parameterization strategies, the Martini 3 models in this work were developed from independently tuned parameters, following the parameterization guidelines for the new Martini 3 force field ${ }^{29}$. These guidelines call for using center of geometry (c.o.g.)-based mapping of atomistic structures, taking into account the respective hydrogen atoms. CG parameters were fit to corresponding atomistic simulations ran with the CHARMM36 force field. In addition, CHARMM36 has proved to be an excellent model for the simulation of phospholipid biological membranes, outperforming competing force fields in terms of experimental data reproducibility ${ }^{65}$. Additionally, the parameterization of the phosphoinositide models for CHARMM36 has been thoroughly described ${ }^{66,67}$ and these parameters have been extensively used for phosphoinositide atomistic simulation studies, both in the presence and absence of divalent cations ${ }^{68-71}$.
With this in mind, we first revisited the parameterization of the core molecule in all phosphoinositide headgroups, the myo-inositol sugar.

\section{myo-Inositol sugar core parameterization}

The bead mapping behind the CG myo-Inositol model followed the Martini 3 rules for 4-1 cyclic/branched fragments, assigning a S-bead for each 2 consecutive ring carbon atoms and their respective hydroxyl groups (Figure 1, myo-Inositol). In our model, carbons 1 and 2, 6 and 5, along with, 4 and 3 correspond to the CG beads $C 1, C 2$ and $C 3$, respectively. This contrasts with the inositol model developed for Martini $2^{30}$, in which the ring was rotated by 1 inositol carbon, meaning that carbons 6 and 1, 4 and 5, and 3 and 2 represented CG beads $C 1, C 2$ and $C 3$, respectively. While both mappings are valid within the Martini guidelines, our mapping should perform better in $\mathrm{PI}(4,5) \mathrm{P}_{2}$ calcium-induced aggregation experiments, as discussed ahead.

To model the bonded parameters for our CG representation, AA simulations of an inositol sugar molecule in water were used as reference (SI Material, figure S2), with initial bonded parameters obtained from the c.o.g.-based mapping of atomistic structures, as mentioned above. To confirm that the c.o.g.-based mapping accurately reflects the atomistic configurations, we compared CG and AA solvent accessible surface areas (SASA). This indicates whether the molecular volume and shape of the CG inositol molecule is representative of the corresponding AA structure. The average SASA values recovered for the single inositol in water systems show a discrepancy of roughly $13 \%$ between the initial CG model and the atomistic parameterization target (Figure 2A). While the current version of Martini 3 systematically underestimates the SASA ${ }^{29}$, a 13\% discrepancy falls beyond a target $10 \%$ error margin. Analyzing the Connolly surfaces (Figure 2, A) we can see that the CG model (red) is not capturing the behavior of the AA model (blue) accurately 

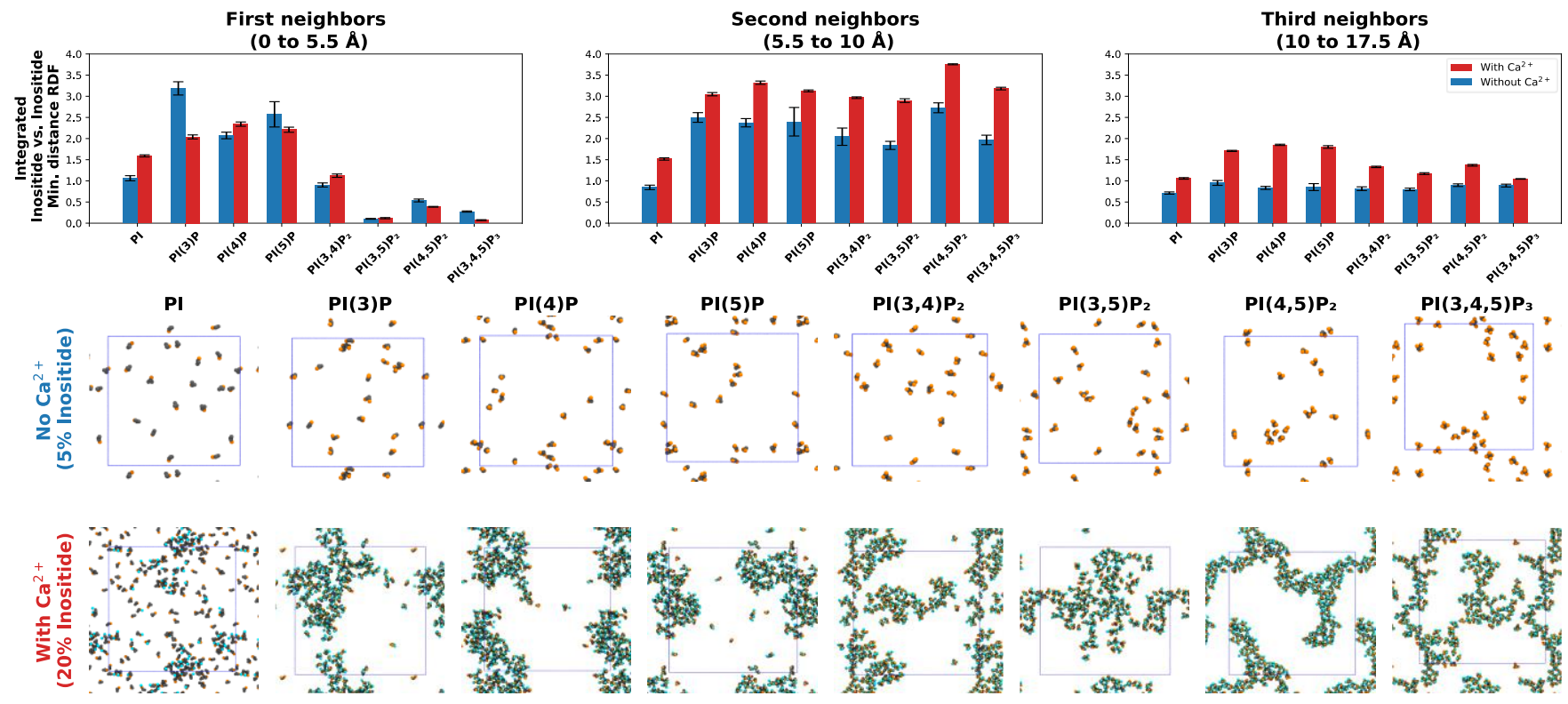

Figure 4. Phosphoinositide aggregation in response to calcium. Integrated inositide vs inositide minimum distance RDFs corresponding to the first, second and third local neighbors, for the 8 inositide lipids. These were obtained from systems in the presence (red) and absence (blue) of calcium at 300K. The full RDFs are included in the SI Material, Figure S14. Final simulation snapshots of the systems are also shown, viewing the top leaflet along the membrane normal, with phosphoinositide lipid headgroups depicted in gray, phosphates in orange, and $\mathrm{Ca}^{2+}$ ions in blue.

enough. This is in part because the hexagon-like inositol ring is now modeled by a triangular arrangement of CG beads, creating a molecular shape mismatch. Additionally, as the hydroxyl groups on the myo-inositol ring are mostly equatorial, they increase the surface area significantly. To improve the model, the CG interparticle distances in the triangular arrangement of inositol were lengthened by $20 \%$. This reduced the discrepancy between the atomistic and CG model to an acceptable $5 \%$, as seen by the recovered SASA values as well as the corresponding Connolly surface (Figure 2A), and was the bonded arrangement used in subsequent inositol parameterization.

myo-Inositol bead type assignment. To assist in our selection of particle types for the myo-inositol model, we set up its octanol/water partition as a target. As a starting point for inositol, beads types ranging from SP4 to SP6 (from less to more polar) were chosen from existing Martini 3 examples representing ethylene glycol moieties. These beads types also roughly correspond to those used by the original Martini 2 inositol model ${ }^{30}$. We ran several octanol/water partition simulations of inositols composed of each bead type, and compared the results against theoretical predictions by XLOGP3-AA ${ }^{72}$, ALOGPS ${ }^{73-75}$ and the ChemAxon Consensus method ${ }^{76,77}$ (Figure 1E). Experimental values could not be found for myo-inositol, possibly because of its strong hydrophilicity, which hampers the use of standard $\log (\mathrm{P})$ determination methods. It is worth noting that while these computational predictions can be quite accurate, at extreme hydrophilicities/hydrophobicities there can often be large disagreements between methods, and between predictions and experiments. The theoretical $\log (\mathrm{P})$ predictors, XLOGP3-AA and ChemAxon yielded $\log (\mathrm{P})$ values of -3.7 and -3.8 respectively, reflecting the strongly hydrophilic character of the myo-inositol ring. ALOGPS yielded a somewhat less hydrophilic $\log (\mathrm{P})$ value of -2.6 . The initially tested CG inositol compositions (all SP4, all SP5 or all SP6 beads) yielded $\log (\mathrm{P})$ values between -4 and -5 , significantly more hydrophilic than any of the predictions. The bead choice closest to the predictions was SP4, the least polar tested type, at a $\log (\mathrm{P})$ of -4.15 . Use of Martini 3 types less polar than SP4, that do not typically represent small diols, was avoided since it may compromise the resulting transferability within the Martini 3 framework.

As a further characterization of the suitability of assigned bead types, we probed inositol self-interaction in solution, comparing CG systems to their atomistic CHARMM36 counterpart in terms of center-of-mass (c.o.m.) inositol-inositol radial distribution functions (RDFs; Figure 2C). In these RDFs, the position of the first CG neighbor matches that of the AA reference. The CG behavior differs, however, in having offset second-neighbor peaks, which was already a feature of the Martini 2 version of the model ${ }^{30}$. The Martini 3 models also differ from the AA reference in having a less pronounced first-neighbor binding. In this aspect, the more polar type (SP6) performs somewhat better.

Given concerns that atomistic models may overestimate self-interaction in sugar molecules ${ }^{78}$, the second osmotic virial coefficient $\left(\mathrm{B}_{22}\right)$ was also determined and compared to experimental values. The $\mathrm{B}_{22}$ coefficient can be used to estimate the self-interaction of sugar molecules, where $B_{22}$ $<0$ indicates net attraction and $\mathrm{B}_{22}>0$ indicates repulsion between molecules. A B $\mathrm{B}_{22}$ value of $0.016 \mathrm{~mol} \cdot \mathrm{L}^{-1}$ has been determined for myo-inositol ${ }^{79}$ (Figure 2, D). CG inositol molecules (Figure 2, D) composed of SP4 beads yielded a B22 value of $0.228 \mathrm{~mol} \cdot \mathrm{L}^{-1}$, indicating a more repulsive behavior than expected. The more polar SP6 beads, however, yielded a value of $-0.098 \mathrm{~mol} \cdot \mathrm{L}^{-1}$, indicating a slightly more attractive behavior than the experimental. Compared to previous Martini 2 models, all Martini 3 models tested here yielded B22 values much closer to the experimental range. Martini 
2 models of similar sugars have typically overestimated their aggregation propensity, recovering values which differ from the experimental values by over one order of magnitude $\left(-1.2 \text { to }-2.0 \mathrm{~mol} \cdot \mathrm{L}^{-1}\right)^{28}$.

Ring-center apolar interaction site. To improve our model, we added an additional Martini 3 Tiny-type (T) particle to the center of the inositol ring - bead $C 4$ - representing the hydrophobic sugar ring core. A TC4 particle was added as a virtual interaction site, constructed in plane as a linear combination of the ring particle positions. With this strategy, the inositol model consisting of the SP4 ring particles and the hydrophobic TC4 virtual site yielded the best matching of the atomistic and experimental behavior in all our tests. While it did not influence the SASA (as the additional particle is buried in the middle of the ring), it led to $\log (\mathrm{P})$ values in much better agreement with theoretical predictors $(\log (\mathrm{P})=-3.74)$, drastically improved inositol self-interaction to near atomistic levels $\left(B_{22}=-0.079 \mathrm{~mol} \cdot \mathrm{L}\right.$ ${ }^{1}$ ), and better approximates the atomistic first-neighbor behavior in Figure 2C. This myo-inositol model was chosen as the basis for subsequent phosphoinositide parameterization.

Besides improving the performance of our model, the added $\mathrm{T}$ particle helps in avoiding underestimating the molecular interaction density. Although $S$ particles are called for when mapping 4-1 branched or cyclic fragments, they must not be overused. As described in the Martini 3 parameterization guidelines ${ }^{29}$, the maximum mismatch should be 1 non-hydrogen atom for each 10 non-hydrogen atoms mapped by CG beads. myo-Inositol has a total of 12 non-hydrogen atoms. Given that a single S particle represents $3 \mathrm{CG}$ mapped non-hydrogen atoms, the $3 \mathrm{~S}$ particles used to map the inositol ring only account for 9 non-hydrogen atoms. The addition of a $\mathrm{T}$ bead in the center of the ring reduces this mismatch and helps avoid interaction density issues.

\section{Phosphoinositide parameterization}

Having finalized the myo-inositol sugar parameterization, we moved on to the phosphoinositide family. Phosphoinositides were parameterized against reference AA counterparts modelled with CHARMM36. The atomistic parameterization target systems for our lipid models were bilayers containing roughly 250 lipids and composed of 95\% POPC and $5 \%$ of the phosphoinositide of interest. This system was chosen over the more common bilayer system containing $100 \%$ of the lipid of interest as it better replicates the phosphoinositide physiological and experimental environments: all phosphoinositides are minority membrane lipids, and most cannot even form stable bilayers at high mol fractions. As a control for lipid overparameterization, a test system composed by a single lipid of interest in a water box is also used. The inclusion of this system reduces parameterization bias for specific membrane-only behavior.

Phosphatidylinositol. The parameterization strategy for PI was i) to bind the inositol model to the phosphodiester phosphate bead from the standard Martini 3 phosphoacylglycerol topology (using 1-palmitoyl-2-oleoyl lipid tail parameters), and then ii) to control the headgroup tilt and rotation by means of appropriate angle and dihedral potentials. All of the inositol headgroup particle types were inherited from the standalone topology, except for the $C 1$ bead, which was changed from SP4 to SP1 to reflect the loss of polarity because one of its hydroxyls now takes part in the phosphodiester bond with the phosphate in the PO4 bead.

It should be noted that the used phosphoacylglycerol topological basis may be subject to optimization in the future, as the full potential offered by Martini 3 is realized. Our parameterization takes that into account, and, for added robustness, only involves headgroup bonded terms with the phosphate and glycerol moieties. This also ensures optimal transferability across Martini 3 phosphoglycerides, which differ only in acyl chain composition.

Regarding the bonded parameters, distances between the headgroup inositol ring beads were adjusted as necessary to account for slight ring torsions originating from intramolecular hydrogen bonds when inserted onto the lipid diacyl glycerol moiety. To control headgroup tilt, an angle potential and 2 bonds were used. The C2-C1-PO4 angle was used to control headgroup tilt with respect to the $P O 4$ bead, while P04-GL1 and P04-GL2 bonds were used to control PO4 tilt with respect to the glycerol backbone. This pair of bonds was used in place of the more common P04-GL1-GL2 angle potential as it allowed us to avoid repulsion between the PO4 and GL2 particles, and thus better represent the atomistic description. With these parameters in place we were able to control headgroup tilt and, importantly, the angle between the inositol headgroup and the membrane surface. To control headgroup rotation, the C3-C2-C1-PO4 and C2C1-P04-GL1 dihedral potentials were put in place. To avoid numerical instabilities and thus allow for longer timesteps, restricted bending potentials were used in place of the regular harmonic angle potentials ${ }^{32}$ when angles that take part in dihedral potentials had distributions close to 0 or $180 \mathrm{de}-$ grees. The remaining lipid parameters are acyl-chain dependent and strictly follow the current Martini 3 lipid parameters. Our approach closely represented the distributions obtained from CG-mapped AA lipid bilayer and lipidin-solution systems (SI Material, Figure S3).

Phosphatidylinositol phosphates. With the core PI lipid fully parameterized, we were able to use it as a building block for the phosphorylated species. Our strategy to add phosphorylations to the core PI lipid was to use a single bond to the inositol ring, one angle potential and one dihedral potential to control the phosphate position relative to the ring plane. When necessary, a weak secondary bond to the ring was employed, to overcome intramolecular repulsions. This was especially useful when parameterizing the polyphosphorylated inositides, where the phosphate beads lie closer to the inositol rings than in the monophosphorylated inositides (this can be seen for $\mathrm{PI}(3,5) \mathrm{P}_{2}$ through the smaller P3-C3-C1 angle compared to the same angle obtained for PI(3)P, Figures S4 and S8). For each phosphate, the main bond was set matching the respective CG-mapped AA distance. When used, the weak secondary bond could be adjusted slightly to allow for the correct angle between ring-phosphate and ring-ring bonds.

In the case of polyphosphorylated inositides, there were instances where two phosphates lay within interaction range of each other. This was often the result of transient interactions between adjacent phosphate groups, mediated by the last phosphate proton. To avoid repulsion and accurately represent the target behavior, a weak bond was put 
A
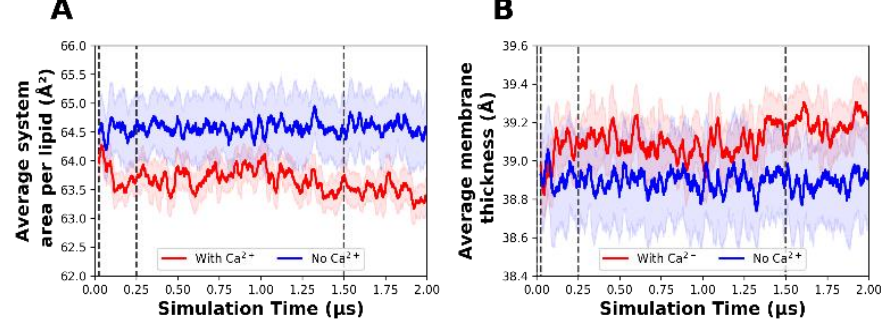

\section{C}

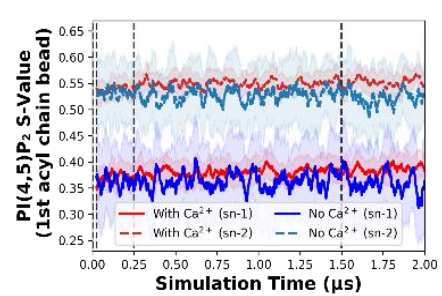

D

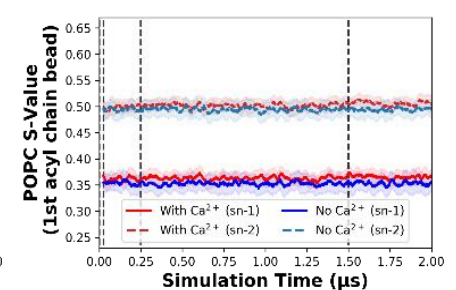

$\mathbf{E}$
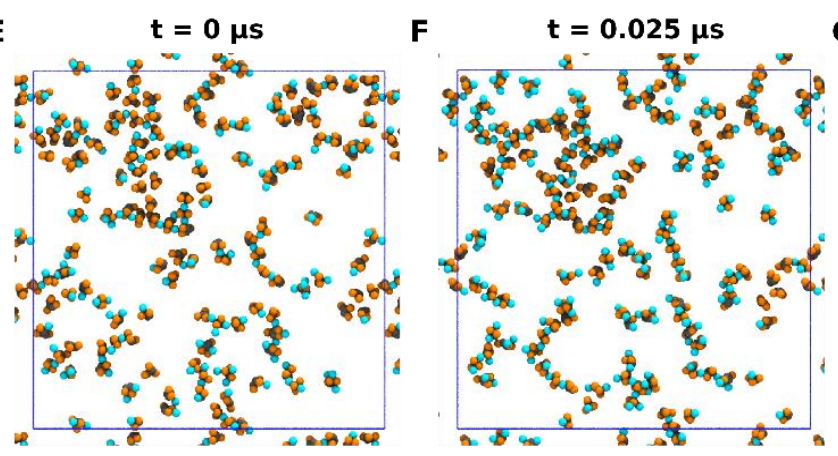

G

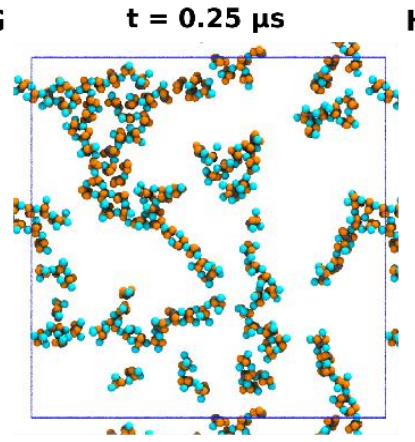

H

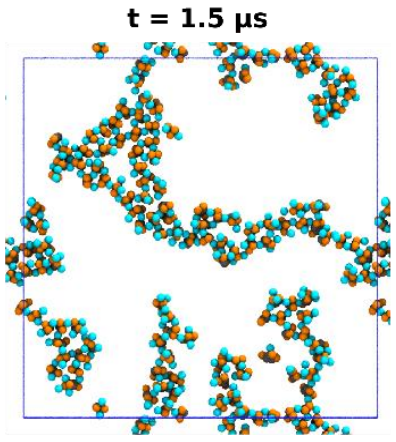

Figure 5. PI(4,5) $\mathrm{P}_{2}$ calcium-induced cluster growth. System area-per-lipid $(\mathrm{A})$, membrane thickness $(\mathrm{B})$, as well as $\mathrm{PI}(4,5) \mathrm{P}_{2}(\mathrm{C})$ and POPC (D) order parameters during the initial calcium-induced cluster growth stages. These were obtained from 20:80 $\mathrm{PI}(4,5) \mathrm{P}_{2}: \mathrm{POPC}$ systems containing $\mathrm{Ca}^{2+}$ at a $2.5: 1 \mathrm{Ca}^{2+}: \mathrm{PI}(4,5) \mathrm{P}_{2}$ ratio at $300 \mathrm{~K}$. The values shown are the running average over the course of 50 frames, with the shaded areas representing the running standard deviation. Simulation snapshots at four timepoints are also shown, with the same viewpoint and coloring as in Figure 4.

in place between them. This was relevant in lipids with adjacent phosphorylations, such as $\mathrm{PI}(3,4) \mathrm{P}_{2}, \mathrm{PI}(4,5) \mathrm{P}_{2}$ and $\operatorname{PI}(3,4,5) \mathrm{P}_{3}$.

As was the case with the phosphodiester, the beads that took part in the phosphomonoester bonds had their polarity reduced from SP4 to SP1 (or from SP1 to SN4a in the case of two phosphomonoester groups bonded to the same bead) to reflect the loss of polarity from the hydroxyl group that is now taking part in the bond.

The addition of phosphate groups induced significant ring torsions and changes to the headgroup tilt angle due to the formation of intramolecular hydrogen bonds with the ring hydroxyls and phosphodiester oxygens ${ }^{33}$. The bonds between the inositol headgroup beads, as well as the angle and dihedral potentials in place were slightly adjusted for each lipid to reflect these dynamics. Our approach correctly represented the distributions obtained from the CG-mapped AA lipid bilayer and lipid in solution systems (SI Material, figures S4 to S10).

Headgroup phosphate protonation state. The protonation state of the phosphodiester group is well defined, being fully deprotonated at physiological $\mathrm{pH}$ (pKa between 1 to $3)^{80}$, and a -1 charge was assigned to it. For phosphatidylinositide mono-phosphates, such as $\mathrm{PI}(4) \mathrm{P}(\mathrm{pKa}(P 4)=6.2)$, the monoprotonated form exists at physiological $\mathrm{pH}$, but the fully deprotonated form is still the dominant population ${ }^{81}$. Headgroup phosphate beads of the mono-phosphorylated lipids were therefore assigned -2 charges.

The protonation behavior of phosphatidylinositol bisphosphates, however, is much more complex. It has been shown that the ionization behavior of phosphoinositides with adjacent phosphate groups cannot be accurately described by a Henderson-Hasselbalch mechanism ${ }^{82}$. Instead, at physiological $\mathrm{pH}$, one proton from the adjacent phosphate group dissociates, while the remaining one is stabilized by being shared between the two vicinal phosphomonoester groups ${ }^{82}$. To add to this complexity, it is likely that a small, fully deprotonated population also exists ${ }^{82}$. With this in mind, the headgroup phosphate beads of the doublephosphorylated lipids with adjacent phosphates $\left(\mathrm{PI}(4,5) \mathrm{P}_{2}\right.$ and $\left.\mathrm{PI}(3,4) \mathrm{P}_{2}\right)$ were set at charge -1.5 each, reflecting the most common charge state (1 phosphate group fully deprotonated with the lasting proton group being mostly shared between the 2 groups) when monodispersed and not interacting with other partners. As for the only double-phosphorylated lipid that does not have adjacent phosphates, $\mathrm{PI}(3,5) \mathrm{P}_{2}$, each of its phosphates presents a mostly well-behaved Henderson-Hasselbach mechanism ${ }^{82}$. At physiological $\mathrm{pH}$, each of its phosphates is split almost evenly into populations of mono-protonated and fully deprotonated species. For this reason, each phosphate bead was set at charge -1.5 , as well, reflecting the most common overall charge state.

$\mathrm{PI}(3,4,5) \mathrm{P}_{3}$, where the three phosphorylations are adjacent, is also a complex case. Its behavior is similar to that of the phosphatidylinositol bisphosphate lipids, with protons being shared between adjacent phosphates ${ }^{82,83}$. As such, the most common charge state at physiological $\mathrm{pH}$, is to have 2 phosphate groups fully deprotonated, with the third phosphate group mono-protonated and sharing its last proton with the adjacent phosphate groups. With this in mind, the $P 3$ and $P 5$ phosphate beads of our CG model of $\mathrm{PI}(3,4,5) \mathrm{P}_{3}$ were set at charge -1.3 and the $P 4$ bead was set at -1.4 to reflect this behavior. The slightly higher charge for the $P 4$ phosphate is in agreement with experimental observations ${ }^{82,83}$.

Phosphate particle types. The -1-charged phosphodiester phosphate was assigned bead type Q5, in line 

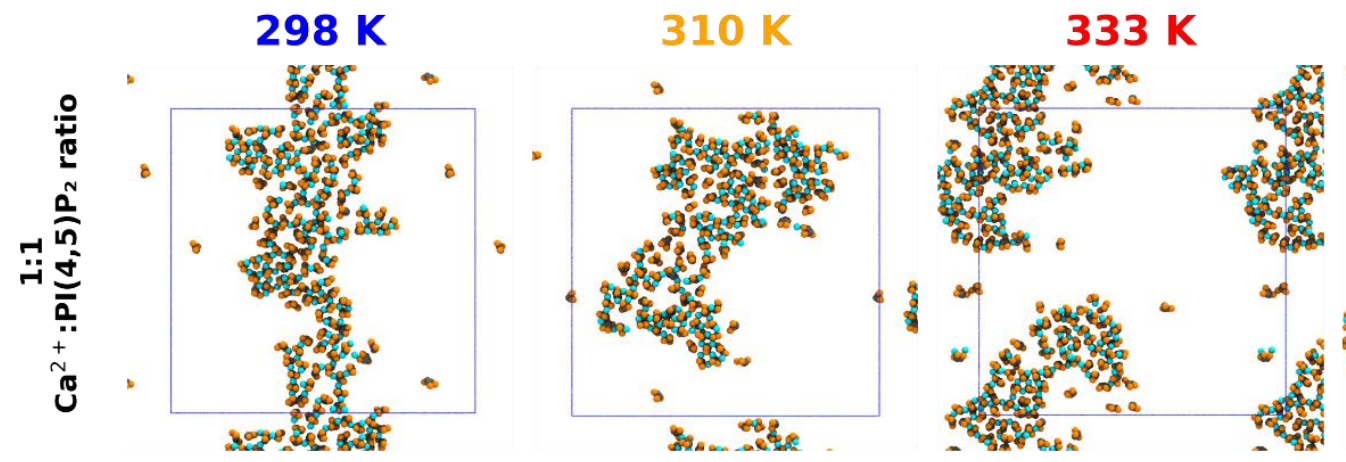

$333 K$ to $298 K$
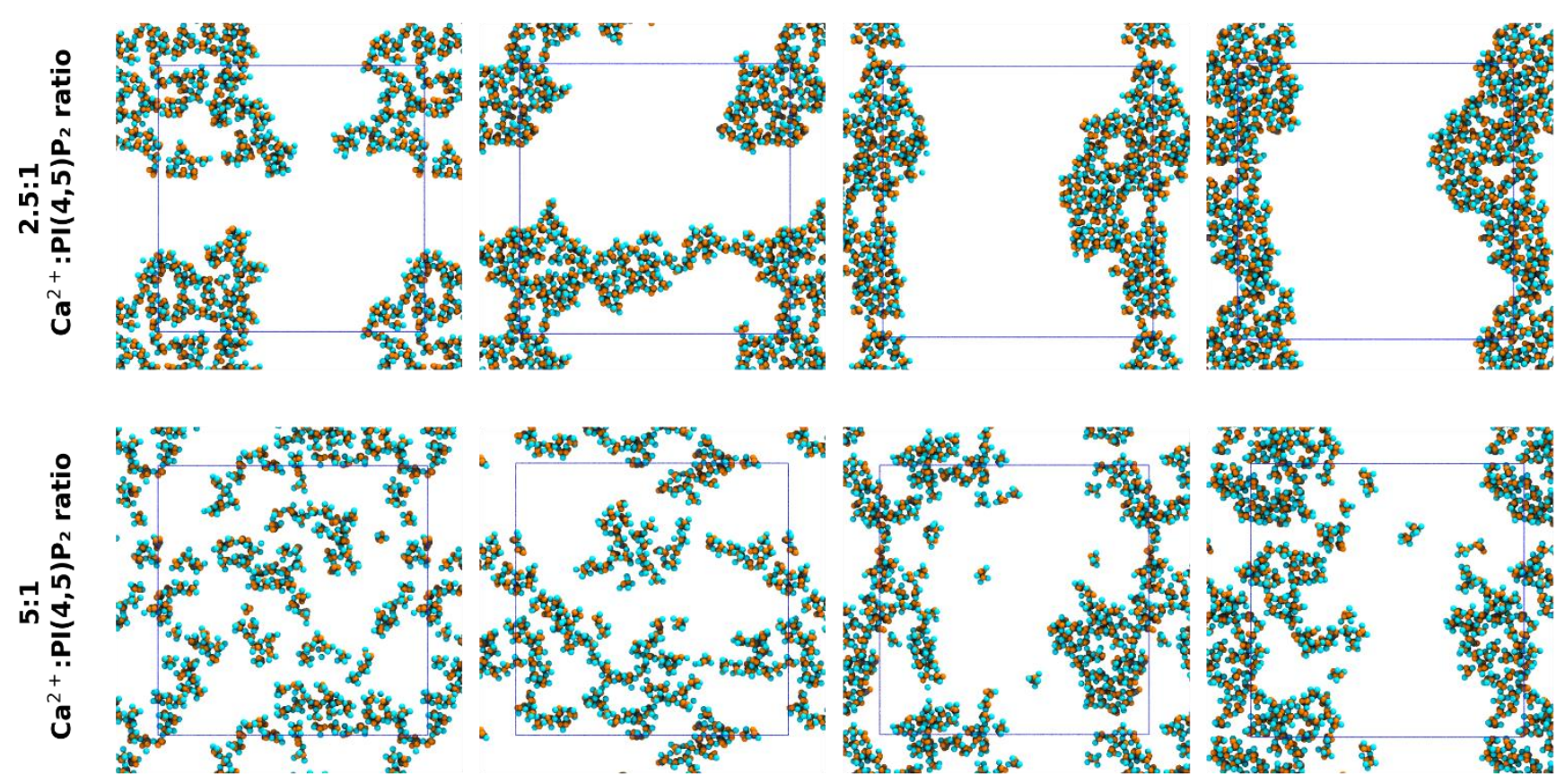

Figure 6. Effects of temperature and $\mathrm{Ca}^{2+}$ concentration on calcium-induced PI(4,5) $\mathrm{P}_{\mathbf{2}}$ clusters. Final simulation snapshots obtained from 20:80 PI(4,5)P2:POPC systems containing $\mathrm{Ca}^{2+}$ at 1:1, 2.5:1, or 5:1 $\mathrm{Ca}^{2+}: \mathrm{PI}(4,5) \mathrm{P}_{2}$ ratios and ran at 298,310 or $333 \mathrm{~K}$. Snapshots from control runs, where the final conformations from the simulations running at $333 \mathrm{~K}$ were used to restart the simulation at $298 \mathrm{~K}$, are also shown. The same viewpoint and component colors as in Figure 4 were employed.

with its charge and with the rest of Martini 3 phospholipid headgroups. For the -2-charged phosphate particles, regular-size D particles - added in Martini $3^{29}$ for divalent hard ions - were used. The -1.5 charged phosphate particles, in $\mathrm{PI}(3,4) \mathrm{P}_{2}, \mathrm{PI}(3,5) \mathrm{P}_{2}$ and $\mathrm{PI}(4,5) \mathrm{P}_{2}$, were modelled as D particles as well, as the last proton is very weakly bonded to the phosphates, conferring significant divalent character. For $\mathrm{PI}(3,4,5) \mathrm{P}_{3}$, however, the $P 3$ and $P 5$ phosphates were modelled as Q5 beads, while the $P 4$ phosphate was modelled as a $\mathrm{D}$ bead. It is worth noting that beads with non-integer charges more anionic than -1 are a parameterization edge case that was not explicitly included in the Martini 3 model. However, Martini 3 was designed to allow the assignment of partial charges when representing charge delocalization over several beads - a situation somewhat analogous to proton sharing between adjacent phosphates.

Apart from the particle types and charges in our CG model, protonation was also taken into consideration when building atomistic references. For the mono-phosphorylated lipids we opted to use fully deprotonated lipids as our target structural reference. In the case of the bis- and tris- phosphorylated species however, atomistic systems contained semi-protonated lipids, where one of the headgroup phosphates is mono-protonated and the other headgroup phosphates are fully deprotonated, in all possible combinations. Our parameterizations were then left as flexible as possible to allow the lipid to visit all possible conformations, allowing for a better representation of the flexibility and variety of conformations that occur in vivo at physiological $\mathrm{pH}$.

\section{Validation}

Membrane behavior. Having fully parameterized the phosphatidylinositol lipid family we then aimed at confirming that the CG lipids accurately represent expected biophysical properties. We started by assessing whether the c.o.g-mapping correctly reflected the atomistic headgroup volumetry, SASA-wise, as was done for myo-inositol. SASA comparisons for each lipid headgroup (from, and including, the phosphodiester phosphate) and their respective atomistic descriptions are shown in Figure 3A. As phosphorylation increases, and with it headgroup size, there is a clear increase in SASA. This is well recovered by our CG models. 
Less phosphorylated species, like PI, do slightly underestimate the SASA in the inositol ring region (Martini 3 tends to underestimate atomistic SASA values ${ }^{29}$ ). This is compensated for by each additional phosphorylation, in that the added individual SASA is slightly larger than that of atomistic phosphates (Figure 3C). As such, the bis and tris-phosphorylated inositides end up having slightly higher SASA than the atomistic model - well within the $10 \%$ accepted margin and all but $\mathrm{PI}(3,4,5) \mathrm{P}_{3}$ within $5 \%$ (Figure $3 \mathrm{~A}$ and $\mathrm{SI}$ Material, Figure S11). For PI, but not for the other phosphoinositides, ring distances were increased to recover the correct SASA behavior, as had been the case for the analogous myo-inositol.

To confirm lipid insertion depth and headgroup surface protrusion when inserted into POPC bilayers, we compared membrane $z$ axis densities between CG systems and AA references (Figure S13). It can be seen that all lipids' phosphodiester group inserts at the same depth as the POPC phosphodiester group. This not only matches the atomistic reference, but also experimental evidence that indicates that this is the preferred depth for PI and PI(4)P84-87. myoInositol rings are slightly more protruded (up to $1 \AA$ ) than in the AA reference, whereas individual headgroup phosphates can protrude up to $2 \AA$ more. Since phosphate-ring distances, as well as most of the bonded terms influencing inositol tilt relative to the glycerol backbone, are well reproduced by our models (Figures S3 to S10) the excessive protrusions may instead result from insufficient headgroupglycerol tilt relative to the lipids' acyl tails. Because we aim for our inositol models to be widely compatible with different tails, in the general scope of Martini 3 lipids, we refrained from introducing specific corrections in this regard.

To quantify the membrane density of each lipid, their area per lipid (ApL) was determined (Figure 3B). Interestingly, unlike the SASA, the ApL measured in atomistic references does not show a clear dependency on headgroup size or phosphorylation. This showcases how phosphoinositides, in general, are able to maintain an ApL similar to that of POPC in spite of a much larger headgroup. Our CG models were able to closely reproduce the atomistic ApL values within a 5\% discrepancy (SI Material, Figure S12).

In all, this analysis shows that the overall biophysical properties of the phosphoinositides parameterized in this work are well represented within the fidelity expected from the Martini 3 force field.

Phosphoinositide aggregation in response to divalent cations. A significant portion of phosphoinositide lateral organization is dependent on the interaction of these lipids with divalent cations. Several phosphoinositides are known to undergo cation-dependent aggregation, forming stable lipid aggregates. These are formed at physiological concentrations of lipid and cations for some of the lipids in the phosphoinositide family and it is likely that some of these are found constitutively clustered in vivo ${ }^{15-17}$. As such, it is of importance that our models accurately represent this aggregation behavior.

We tested the response of each phosphoinositide to the presence of calcium in a 2.5:1 $\mathrm{Ca}^{2+}$ :phosphoinositide ratio (Figure 4). These systems were built at a 20:80 inositide:POPC ratio to mimic the exceptionally high inositide concentrations in the local vicinity of phosphoinositide domains. In the systems where calcium is included, phosphoinositide headgroup phosphates are assigned a charge of -2 and their bead type changed to $\mathrm{D}$, when applicable. This mimics the displacement of the last phosphate proton when calcium binds phosphoinositide headgroups. To test phosphoinositide aggregation in the absence of calcium, we employed membranes at a 5:95 inositide:POPC ratio, mimicking monodisperse phosphoinositide conditions.

The analysis of inositide vs inositide RDFs obtained from the minimum distance between headgroups, combined with the visual analysis of the structures obtained, proved useful in characterizing, and comparing the domains formed by each inositide species. In general, the first-neighbor peak in these RDFs represent direct inositide-inositide contacts. The second-neighbor peaks represent inositide-inositideinositide contacts, but, in the case of systems with calcium, they mostly represent calcium-linked inositide dimers (i.e. inositide- $\mathrm{Ca}^{2+}-$ inositide contacts). Further peaks represent larger contact chains.

In the absence of divalent cations none of the parameterized PI lipids self-aggregate (Figure 4). This is in agreement with most experimental findings which point at PIs being monodisperse in fluid bilayers ${ }^{15,17,88}$. The monophosphorylated inositides showed the most first-neighbor contacts compared to the bis- and tris- phosphorylated lipids. This hints that the additional bulky phosphates and associated negative charge in polyphosphorylated inositides, either increases repulsion between the monodisperse lipids or leads to more inefficient stacking of the headgroups, or both. Interestingly, the mono-phosphorylated inositides also showed increased first-neighbor contacts when compared to PI, showing that the added repulsion of the single headgroup phosphate can be compensated for by the establishing of additional interactions.

In the presence of calcium, however, all inositol lipids except for PI quickly undergo very significant cation-induced aggregation. This can be clearly seen, both through the final simulation snapshots as well as through the integrated RDFs (Figure 4). While PI did not form calcium-induced clusters, it led to some adsorption of calcium at the phosphodiester depth, which led to the formation of PI dense areas, with no defined structure. This can be clearly seen in the simulation snapshots and integrated RDF peaks (Figure 4).

We see no significant calcium-induced increase in the density of first neighbors among the phosphorylated lipids, but we see a large increase in density of second and third neighbors, for all inositides. $\mathrm{PI}(4,5) \mathrm{P}_{2}$ formed the densest calcium-induced nanodomains, with barely any POPC mixed in with the inositide. This can be clearly seen by the increased density of second neighbors. These domains are irregularly shaped, with a lobed morphology and a fairly organized stacking of the inositol headgroups (Figure S17).

The mono-phosphorylated inositides, formed less dense, irregular domains that contained a significant amount of POPC lipids within. These domains also showed much more irregular stacking of the headgroups (Figure S17). The rough circular shape of these domains led to a significant increase of density of third neighbors when compared to the other inositides. For $\mathrm{PI}(3,4) \mathrm{P}_{2}, \mathrm{PI}(3,5) \mathrm{P}_{2}$ and $\mathrm{PI}(3,4,5) \mathrm{P}_{3}$, 
A

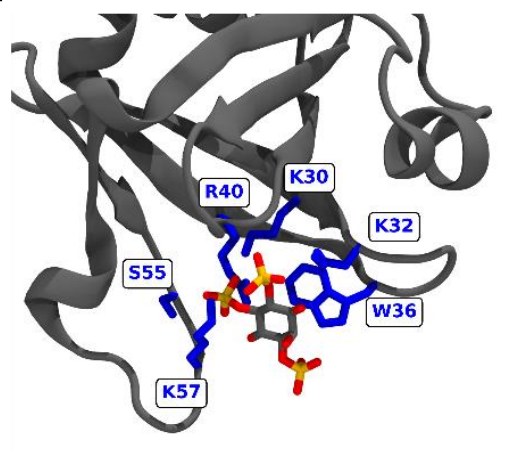

B

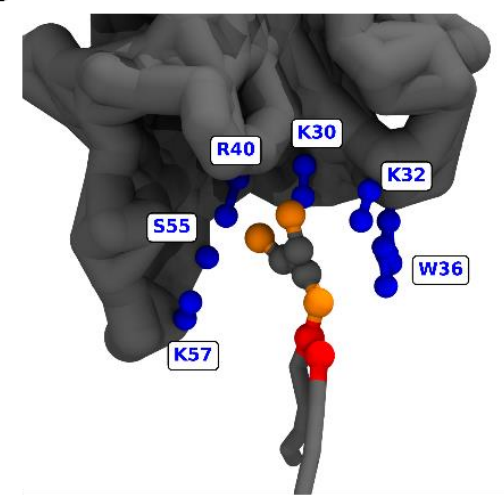

C

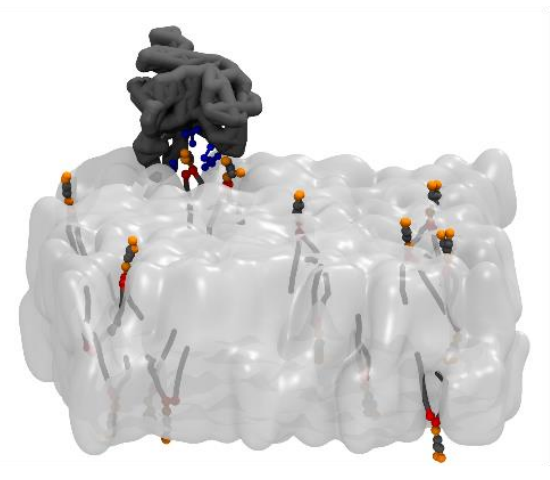

Figure 7. PI(4,5)P $P_{2}$ recognition and binding by PLC 1 PH. PLC $\delta 1$ PH domain bound to inositol 1,4,5-trisphosphate as determined by X-ray diffraction (PDB: $1 \mathrm{MAI}^{58}$ ) (A). Martini 3 CG PLC $\delta 1$ PH domain bound to PI $(4,5) \mathrm{P}_{2}(\mathrm{~B})$. Snapshot of the CG membrane system showcasing $\mathrm{PH}$ domain membrane recognition and $\mathrm{PI}(4,5) \mathrm{P}_{2}$ binding (C). PH domains are depicted in gray, with the $\mathrm{PI}(4,5) \mathrm{P}_{2}$ binding pocket amino acid residues explicitly shown in blue. POPC is shown as the translucent surface.

domains formed with density similar to the mono-phosphorylated cases, although apparently less localized. While $\mathrm{PI}(3,5) \mathrm{P}_{2}$ and $\mathrm{PI}(3,4,5) \mathrm{P}_{3}$ domains show very little headgroup organization, $\mathrm{PI}(3,4) \mathrm{P}_{2}$ domains are slightly more organized, as was the case with $\mathrm{PI}(4,5) \mathrm{P}_{2}$ (Figure S17).

The observed CG-simulated behavior is roughly in agreement with available experimental results. Calcium-induced clusters of $\mathrm{PI}(4,5) \mathrm{P}_{2}$ and $\mathrm{PI}(3,5) \mathrm{P}_{2}$ have been observed in model membranes at physiological concentrations of both calcium and lipid, with $\mathrm{PI}(4,5) \mathrm{P}_{2}$ inducing larger and likely more stable clusters than $\mathrm{PI}(3,5) \mathrm{P}_{2}{ }^{16}$ (the behavior of $\mathrm{PI}(4,5) \mathrm{P}_{2}$ is discussed and explored in greater detail below). This agrees with the behavior of our Martini 3 bis-phosphorylated inositides. On the other hand, when the monophosphorylated PI(4)P was tested in the same experimental conditions, no calcium-induced clusters were observed ${ }^{16}$. However, mono-phosphorylated PI species can co-cluster with bis-phosphorylated inositides such as $\mathrm{PI}(4,5) \mathrm{P}_{2}$, showing that they do have some clustering propensity ${ }^{17}$. We could not find any experimental evidence on cation-induced aggregation of $\mathrm{PI}(3,4,5) \mathrm{P}_{3}$; however, it is believed that it likely does ${ }^{89}$. As for PI, there is no experimental evidence for its cation-induced clustering in model membranes, and it has been shown that it does not co-cluster with other inositide lipids ${ }^{17}$. This is in agreement with our observation of no significant PI aggregation in the presence of calcium.

Phosphoinositide aggregation and choice of mapping. In our models, the inositol sugar mapping is rotated by 1 atomistic carbon when compared to the Martini 2 inositol mapping, i.e. carbons 1 and 2, 6 and 5, along with, 4 and 3 correspond to the CG beads $C 1, C 2$ and $C 3$ respectively. This was done to simplify the modelling of $\mathrm{PI}(4,5) \mathrm{P}_{2}$, keeping it symmetric with the headgroup phosphate beads bonded to separate inositol beads. While both mappings are valid, this should slightly improve $\mathrm{PI}(4,5) \mathrm{P}_{2}$ self-interactions, as well as headgroup packing in cation-induced nanodomains.

In comparison to our Martini 3 models, the Martini 2 models lead to much more dramatic aggregation (Figure S15), with every phosphoinositide undergoing significant calcium-induced clustering. This more intense aggregation is likely to be the combined result of several effects: overly loose phosphoinositide conformational dynamics, less precise cation-phosphate interactions, and overestimated phosphoinositide self-interactions. The drastic increase in first-neighbor density, and the highly organized headgroup structure of the domains formed with the Martini 2 models, hint that overestimated self-interactions are likely the main driver. Such overestimation is one of the main aspects that Martini 3 mitigates ${ }^{20,29}$.

Characterization of $\mathrm{PI}(4,5) \mathrm{P}_{2}$ calcium-induced clusters. $\mathrm{PI}(4,5) \mathrm{P}_{2}$ has been shown to tightly bind both calcium and magnesium through strong electrostatic interactions between the negatively charged headgroup phosphates and the divalent ions. These divalent cations can influence $\mathrm{PI}(4,5) \mathrm{P}_{2}$ lateral organization dramatically, inducing the formation of nanodomains. Cation-induced nanodomains have been observed in model membranes at physiological concentrations of both lipid and cation using fluorescence spectroscopy methods ${ }^{15-17}$ and $\mathrm{AFM}^{90}$. $\mathrm{PI}(4,5) \mathrm{P}_{2}$ nanodomains have also been observed in PC12 cells using single-molecule imaging techniques ${ }^{91}{ }^{92}$. Additionally, it has been shown in fluorescence correlation spectroscopy experiments carried out in Rat1 fibroblasts and HEK cells, that the diffusion of $\mathrm{PI}(4,5) \mathrm{P}_{2}$ is significantly slower than expected for free phospholipids, concluding that approximately two-thirds of $\mathrm{PI}(4,5) \mathrm{P}_{2}$ in the inner leaflet of the plasma membrane is somehow sequestered ${ }^{93}$.

Our Martini $3 \mathrm{PI}(4,5) \mathrm{P}_{2}$ CG model can undergo the formation of these cation-induced clusters in a qualitatively accurate manner, and, in contrast to the other lipids, form fairly organized structures. There are no experimental studies on the structural organization of $\mathrm{PI}(4,5) \mathrm{P}_{2}$ within these nanodomains, and the little structural evidence available comes from atomistic MD studies that do not actually form fully assembled $\mathrm{PI}(4,5) \mathrm{P}_{2}$ nanodomains, but rather smaller aggregates, due to either system size, timescale or force field limitations ${ }^{71}$.

Figure 5 depicts the formation dynamics of $\mathrm{PI}(4,5) \mathrm{P}_{2}$ clusters. At $\mathrm{t}=0$, after minimization and relaxation, a fair amount of calcium has already adsorbed onto the membrane surface. A continued decrease in ApL with increasing calcium adsorption is observed over simulation time. Also at $\mathrm{t}=0 \mathrm{we}$ 
A

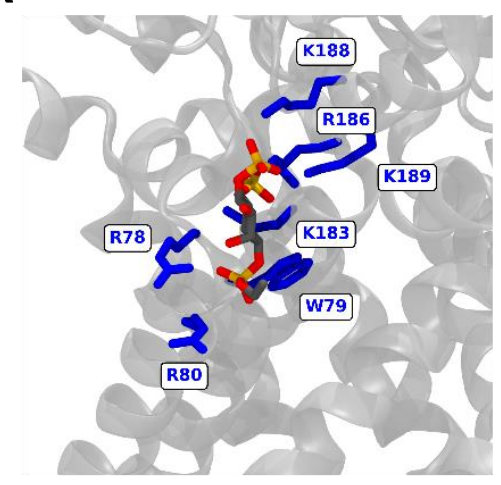

C

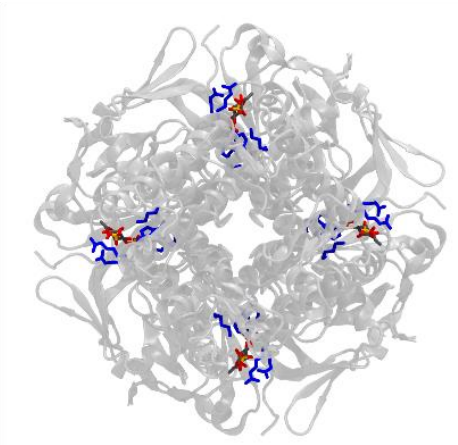

B

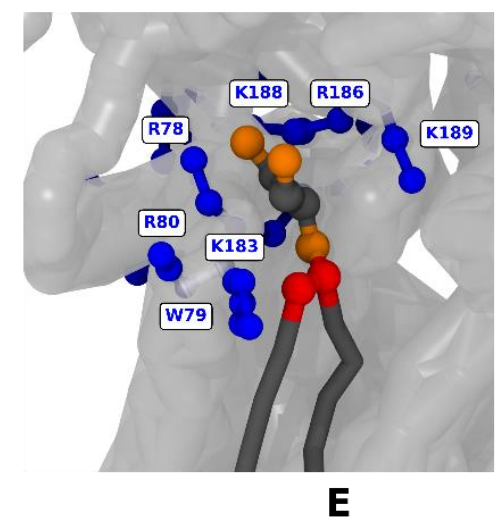

Figure 8. PI(4,5) $\mathrm{P}_{2}$ recognition and binding to the Kir2.2 channel. $\mathrm{PI}(4,5) \mathrm{P}_{2}$ binding site $(\mathrm{A})$ and top view $(\mathrm{C})$ of Kir2.2 bound to $\mathrm{PI}(4,5) \mathrm{P}_{2}$ as determined by $\mathrm{x}$-ray diffraction (PDB: $\left.6 \mathrm{M} 84^{59}\right)$. $\mathrm{PI}(4,5) \mathrm{P}_{2}$ binding site $(\mathrm{B})$, top view $(\mathrm{D})$, and $\mathrm{PI}(4,5) \mathrm{P}_{2}$ density map of Martini 3 Kir2.2 bound to PI(4,5) $\mathrm{P}_{2}$. Kir2.2 channels are depicted in grey, with the PI(4,5) $\mathrm{P}_{2}$ binding pocket amino acid residues explicitly shown in blue. $\mathrm{PI}(4,5) \mathrm{P}_{2}$ densities are shown in red volume maps, at an isovalue corresponding to at least a $3 \%$ occupancy.

can already see the formation of the first aggregates, with $\mathrm{PI}(4,5) \mathrm{P}_{2}$ assembling into small filaments.

After 25 ns, the filament-like clusters continue to grow, and, at 250 ns large 10 lipid filament structures are observed alongside a significant decrease in APL, and an increase in membrane thickness. At this point, these structures are in qualitative agreement with those found by Han et $a .^{94}$ who, in spite of a significantly different setup (monolayers composed of $\left.100 \% \operatorname{PI}(4,5) \mathrm{P}_{2}\right)$, observed the formation of stringlike calcium-induced clusters. Han et al. also detect a decrease in ApL, in agreement with our findings. Other authors have also reported similar cluster structures and ApL responses, using atomistic bilayer systems with low $\mathrm{PI}(4,5) \mathrm{P}_{2}$ concentrations ${ }^{71}$.

After $1.5 \mu \mathrm{s}$, the $\mathrm{PI}(4,5) \mathrm{P}_{2}$ filament-like structures begin to coalesce and form the denser, lobed structures. With the formation of these structures, we also begin to see a slight increase in the acyl-chain order of both PI $(4,5) \mathrm{P}_{2}$ and POPC. The ordering of $\mathrm{PI}(4,5) \mathrm{P}_{2}$ acyl-chains is also in agreement with experimental data ${ }^{95}$. At longer timescales, the domains continue to coalesce (Figure $\left.4, \mathrm{PI}(4,5) \mathrm{P}_{2}\right)$ and these effects on the membrane properties are either maintained or further magnified by the increase in cluster density (SI Material, Figure S18).

Finally, we tested how the formation of $\mathrm{PI}(4,5) \mathrm{P}_{2}$ domains responds to varying temperature and $\mathrm{Ca}^{2+}: \mathrm{PI}(4,5) \mathrm{P}_{2}$ ratio (Figure 6). Temperature, up to the tested $333 \mathrm{~K}$, does not appear to significantly impact lipid organization. While we observe larger clusters at higher temperatures, it looks as if it is simply the result of the speed-up kinetics (i.e. higher lipid diffusion coefficient). To confirm this, the final conformations from the systems run at $333 \mathrm{~K}$ were used to re-start the simulations at $298 \mathrm{~K}$. No significant changes to the clusters were observed from lowering the temperature. Both $\mathrm{PI}(4,5) \mathrm{P}_{2}-\mathrm{PI}(4,5) \mathrm{P}_{2}$ and $\mathrm{PI}(4,5) \mathrm{P}_{2}-\mathrm{POPC}$ RDFs confirm these observations (SI Material, Figure S19), which are also in agreement with experimental results ${ }^{95}$.

Calcium concentration, however, does influence the formation of $\mathrm{PI}(4,5) \mathrm{P}_{2}$ domains in a more complex manner. At $1: 1 \mathrm{Ca}^{2+}: \mathrm{PI}(4,5) \mathrm{P}_{2}$ ratio, the clusters formed are not very dense, with some room for other lipids to be incorporated. When the $\mathrm{Ca}^{2+}: \mathrm{PI}(4,5) \mathrm{P}_{2}$ ratio is increased to 2.5 , clusters become much denser and tighter packed. However, a further increase in the $\mathrm{Ca}^{2+}: \mathrm{PI}(4,5) \mathrm{P}_{2}$ ratio to 5 , causes clusters to become disrupted; while some $\mathrm{PI}(4,5) \mathrm{P}_{2}$ filament-like structures are still formed, they are sparsely interconnected and not very condensed, especially at temperatures below $333 \mathrm{~K}$. At high calcium concentrations, $\mathrm{PI}(4,5) \mathrm{P}_{2}$ headgroups no longer gain from sharing cations, as there are enough ions to fully screen the lipids without bridging lipids together.

$\mathrm{PI}(4,5) \mathrm{P}_{2}$ recognition and binding by proteins. A crucial area of phosphoinositide research is their interaction with proteins. Phosphoinositide-protein interactions are at the base of their downstream cellular signaling, and, as such, our models must correctly replicate the experimental behavior. To test this, we probed the recognition and binding of our Martini 3 model of $\mathrm{PI}(4,5) \mathrm{P}_{2}$ by 2 canonical 
$\mathrm{PI}(4,5) \mathrm{P}_{2}$-binding proteins, the pleckstrin homology $(\mathrm{PH})$ domain from phospholipase $\mathrm{C} \delta$ (PLC $\delta 1)$ and an inward rectifier potassium (Kir2.2) channel.

The isolated PLC $1 \mathrm{PH}$ domain was found to bind to $\mathrm{PI}(4,5) \mathrm{P}_{2}$ with high affinity and specificity ${ }^{96,97}$. In fact, these studies provided the first demonstrations of specific PI recognition by a $\mathrm{PH}$ domain and showed how binding domains recognize specific phosphoinositides in membranes $^{98}$. PLC $\delta 1 \mathrm{PH}$ domains are still to this day used as excellent protein models to study $\mathrm{PI}(4,5) \mathrm{P}_{2}$-protein interactions and $\mathrm{PI}(4,5) \mathrm{P}_{2}$ organization.

To test whether $\mathrm{PI}(4,5) \mathrm{P}_{2}$ recognition by the $\mathrm{PH}$ domain is in agreement with previous findings, we built membrane systems at a 5:95 $\mathrm{PI}(4,5) \mathrm{P}_{2}: \mathrm{POPC}$ ratio and placed a $\mathrm{PH}$ domain in the corner of the simulation box. Over the course of the simulation, the PH domain correctly captured its binding orientation and probed the surface of the membrane, eventually recognizing and binding a $\mathrm{PI}(4,5) \mathrm{P}_{2}$ lipid in its binding pocket (Figure 7C). The interactions established by the Martini 3 model (Figure 7B), are in agreement with those established by the X-ray structure of the $\mathrm{PH}$ domain bound to the soluble headgroup of $\mathrm{PI}(4,5) \mathrm{P}_{2}{ }^{58}$ (Figure $7 \mathrm{~A}$ ), as well as those observed in previous Martini 2 simulation studies $^{61,99}$. Most of the amino acid contacts that were observed in the X-ray structure were also observed in our Martini 3 model (K30, K32, W36, R40, S55 and K57), as well as a very similar binding pose (Figure $7 \mathrm{~A}$ and $\mathrm{B}$ ). These findings point at a successful $\mathrm{PH}$ domain recognition and binding of PI $(4,5) \mathrm{P}_{2}$.

Kir channels are tetrameric transmembrane potassium channels, composed of identical subunits. These channels, were found to be regulated by several lipid species, especially $\mathrm{PI}(4,5) \mathrm{P}_{2}$ which was found to activate mammalian Kir channels ${ }^{100,101}$. Resolved crystal structures of Kir channels with bound $\mathrm{PI}(4,5) \mathrm{P}_{2}{ }^{59}$, as well as previous Martini 2 simulation studies ${ }^{23,62}$, show a binding pocket from which the $\mathrm{PI}(4,5) \mathrm{P}_{2}$ headgroup may interact with both the transmembrane and cytoplasmic domain, favoring the open conformation.

To test $\mathrm{PI}(4,5) \mathrm{P}_{2}$ binding to Kir channels, an asymmetric membrane system was built, composed of fully POPC on the upper leaflet and of a 5:95 POPC: PI(4,5) $\mathrm{P}_{2}$ ratio on the lower leaflet. A Kir2.2 channel was positioned roughly as described in previous simulation studies ${ }^{23,62}$. Over the course of the simulation, $\mathrm{PI}(4,5) \mathrm{P}_{2}$ lipids diffused freely and converged upon a site common to all subunits (Figure 8D and E) which overlaps with the $\mathrm{PI}(4,5) \mathrm{P}_{2}$ binding site detected by X-ray crystallography ${ }^{59}$ (Figure 8C). Indeed, $\mathrm{PI}(4,5) \mathrm{P}_{2}$ lipids were found bound to the described Kir binding site (Figure 8B), and established amino acid contacts (K189, K186, K188, K183, R78, R80, W79) which are in agreement with those observed in the resolved X-ray structure $^{59}$ as well as in previous Martini 2 studies ${ }^{23,62}$.

The recognition and binding behavior, observed with both the PLC $\delta 1$ PH domain and the Kir2.2 channel, shows that the Martini $3 \mathrm{PI}(4,5) \mathrm{P}_{2}$ model is correctly interacting with proteins and replicating experimental findings. While other inositide species were not tested, it is safe to assume, given the Martini chemical building block approach, that they should also be well behaved and accurately replicate experimental interactions.

\section{Conclusion}

In this work we successfully develop and validate Martini 3 CG topologies for inositol and 8 phosphoinositides. More than a simple version update from existing Martini 2 models, these are models developed independently from their Martini 2 counterparts, with greater accuracy and expanded application scope to include, among others, accurate reproduction of cation-mediated phosphoinositide aggregation. This enabled a thorough characterization of calcium-induced $\mathrm{PI}(4,5) \mathrm{P}_{2}$ clusters, which showed biophysical and structural properties in agreement with available AA and experimental evidence.

The models we develop here were built and tested atop the phosphoacylglycerol backbone initially put forth with Martini 3. Should that model be refined, phosphoinositide behavior could be affected. The parameterization strategy we lay out here can then be employed again to adjust the phosphoinositide models. In fact, force field development is a constantly ongoing effort, as attested to by the recent release of the significantly improved Martini 3 - even as Martini 2 enjoys wide application as successful use. Our phosphoinositide models should be no exception, and we look forward to revisiting their parameterization as CG methodologies evolve, as more accurate atomistic models are developed, and as new experimental data become available.

\section{ASSOCIATED CONTENT}

Extended Methods and Materials providing details behind the simulation analysis, as well as additional analysis results on phosphoinositide parameterization, calcium-induced aggregation and characterization of $\mathrm{PI}(4,5) \mathrm{P}_{2}$ calcium-induced clusters. martini_v3.0_phosphoinositides_v1.0.itp is a molecule topology description file, in text format compatible with the GROMACS software, containing the CG parameters for the Martini 3 phosphoinositide lipid models developed in this study, linked to several acyl-chain profiles, and the soluble inositol and phosphoinositide headgroup models derived from the lipid topologies.

\section{AUTHOR INFORMATION}

\section{Corresponding Author}

Manuel N. Melo - Instituto de Tecnologia Química e Biológica António Xavier, Universidade Nova de Lisboa, Oeiras 2780157, Portugal; orcid.org/0000-0001-6567-0513 ; m.n.melo@itqb.unl.pt

\section{Author Contributions}

L.B.A., P.C.T.S and M.N.M. designed the models. All authors designed the simulation/validation systems. L.B.A. and M.N.M. designed the analyses. L.B.A. performed simulations and analyses. All authors contributed to manuscript writing and revision and have given approval to the final version of the manuscript.

\section{Notes}

The authors declare no competing financial interest. 


\section{ACKNOWLEDGMENTS}

The authors thank S. Thallmair for critical discussion during parameter development. L.B.A. thanks the Medical Biochemistry and Biophysics Doctoral Program (M2B-PhD) and Fundação para a Ciência e a Tecnologia, I.P. (FCT) for PhD fellowship PD/BD/137492/2018. M.N.M. also acknowledges FCT for funding Project MOSTMICRO-ITQB, with references UIDB/04612/2020 and UIDP/04612/2020.

\section{REFERENCES}

(1) Viaud, J.; Mansour, R.; Antkowiak, A.; Mujalli, A.; Valet, C.; Chicanne, G.; Xuereb, J. M.; Terrisse, A. D.; Séverin, S.; Gratacap, M. P.; Gaits-Iacovoni, F.; Payrastre, B. Phosphoinositides: Important Lipids in the Coordination of Cell Dynamics. Biochimie 2016, 125, 250-258.

(2) Balla, T. Phosphoinositides: Tiny Lipids with Giant Impact on Cell Regulation. Physiol. Rev. 2013, 93 (3), 1019-1137.

(3) Höning, S.; Ricotta, D.; Krauss, M.; Späte, K.; Spolaore, B.; Motley, A.; Robinson, M.; Robinson, C.; Haucke, V.; Owen, D. J. Phosphatidylinositol-(4,5)-Bisphosphate Regulates Sorting Signal Recognition by the Clathrin-Associated Adaptor Complex AP2. Mol. Cell 2005, 18 (5), 519-531.

(4) Posor, Y.; Eichhorn-Grünig, M.; Haucke, V. Phosphoinositides in Endocytosis. Biochimica et Biophysica Acta-Molecular and Cell Biology of Lipids. Elsevier B.V. June 1, 2015, pp 794-804.

(5) Levin, R.; Grinstein, S.; Schlam, D. Phosphoinositides in Phagocytosis and Macropinocytosis. Biochimica et Biophysica Acta - Molecular and Cell Biology of Lipids. Elsevier B.V. June 1, 2015, pp 805-823.

(6) Hilgemann, D. W.; Feng, S.; Nasuhoglu, C. The Complex and Intriguing Lives of PIP2 with Ion Channels and Transporters. Sci. STKE 2001, 2001 (111), re19.

(7) Dickson, E. J.; Hille, B. Understanding Phosphoinositides: Rare, Dynamic, and Essential Membrane Phospholipids. Biochem. J. 2019, 476 (1), 1-23.

(8) Hille, B.; Dickson, E. J.; Kruse, M.; Vivas, O.; Suh, B. C. Phosphoinositides Regulate Ion Channels. Biochimica et Biophysica Acta - Molecular and Cell Biology of Lipids. Elsevier B.V. June 1, 2015, pp 844-856.

(9) Tsujita, K.; Itoh, T. Phosphoinositides in the Regulation of Actin Cortex and Cell Migration. Biochimica et Biophysica Acta - Molecular and Cell Biology of Lipids. Elsevier B.V. June 1, 2015, pp 824-831.

(10) Fiume, R.; Faenza, I.; Sheth, B.; Poli, A.; Vidalle, M. C.; Mazzetti, C.; Abdul, S. H.; Campagnoli, F.; Fabbrini, M.; Kimber, S. T.; Mariani, G. A.; Xian, J.; Marvi, M. V.; Mongiorgi, S.; Shah, Z.; Divecha, N. Nuclear Phosphoinositides: Their Regulation and Roles in Nuclear Functions. Int. J. Mol. Sci. 2019, 20 (12), 2991.

(11) Raghu, P.; Joseph, A.; Krishnan, H.; Singh, P.; Saha, S. Phosphoinositides: Regulators of Nervous System Function in Health and Disease. Frontiers in Molecular Neuroscience. Frontiers Media S.A. August 23, 2019, p 208.

(12) Bunney, T. D.; Katan, M. Phosphoinositide Signalling in Cancer: Beyond PI3K and PTEN. Nature Reviews Cancer. Nature Publishing Group May 2010, pp 342-352.

(13) Thapa, N.; Tan, X.; Choi, S.; Lambert, P. F.; Rapraeger, A. C.; Anderson, R. A. The Hidden Conundrum of Phosphoinositide Signaling in Cancer. Trends in Cancer. Cell Press July 1, 2016, pp 378-390.

(14) Irvine, R. F. Thematic Review Series: Living History of Lipids: A Short History of Inositol Lipids. Journal of Lipid Research. American Society for Biochemistry and Molecular Biology Inc. November 1, 2016, pp 1987-1994.

(15) Sarmento, M. J.; Coutinho, A.; Fedorov, A.; Prieto, M.; Fernandes, F. Ca2+ Induces PI(4,5)P2 Clusters on Lipid Bilayers at Physiological PI(4,5)P2 and Ca2+ Concentrations. Biochim. Biophys. Acta 2014, 1838 (3), 822-830.

(16) Sarmento, M. J.; Coutinho, A.; Fedorov, A.; Prieto, M.; Fernandes, F. Membrane Order Is a Key Regulator of Divalent Cation-Induced Clustering of $\mathrm{PI}(3,5) \mathrm{P} 2$ and $\mathrm{PI}(4,5) \mathrm{P} 2$.
Langmuir 2017, 33 (43), 12463-12477.

(18) Ingólfsson, H. I.; Melo, M. N.; Van Eerden, F. J.; Arnarez, C.; Lopez, C. A.; Wassenaar, T. A.; Periole, X.; De Vries, A. H.; Tieleman, D. P.; Marrink, S. J. Lipid Organization of the Plasma Membrane. J. Am. Chem. Soc. 2014, 136 (41), 14554-14559.

(19) Marrink, S. J.; Risselada, H. J.; Yefimov, S.; Tieleman, D. P.; De Vries, A. H. The MARTINI Force Field: Coarse Grained Model for Biomolecular Simulations. J. Phys. Chem. B 2007, 111 (27), 7812-7824.

(20) Alessandri, R.; Souza, P. C. T.; Thallmair, S.; Melo, M. N.; de Vries, A. H.; Marrink, S. J. Pitfalls of the Martini Model. J. Chem. Theory Comput. 2019, 15 (10), 5448-5460.

(21) Marrink, S. J.; Corradi, V.; Souza, P. C. T.; Ingólfsson, H. I.; Tieleman, D. P.; Sansom, M. S. P. Computational Modeling of Realistic Cell Membranes. Chemical Reviews. American Chemical Society May 8, 2019, pp 6184-6226.

(22) Ingólfsson, H. I.; Carpenter, T. S.; Bhatia, H.; Bremer, P. T.; Marrink, S. J.; Lightstone, F. C. Computational Lipidomics of the Neuronal Plasma Membrane. Biophys. J. 2017, 113 (10), 2271-2280.

(23) Stansfeld, P. J.; Hopkinson, R.; Ashcroft, F. M.; Sansom, M. S. P. PIP 2 -Binding Site in Kir Channels: Definition by Multiscale Biomolecular Simulations. Biochemistry 2009, 48 (46), 10926-10933.

(24) Hansen, S. B.; Tao, X.; MacKinnon, R. Structural Basis of PIP2 Activation of the Classical Inward Rectifier K+ Channel Kir2.2. Nature 2011, 477 (7365), 495-498.

(25) Stark, A. C.; Andrews, C. T.; Elcock, A. H. Toward Optimized Potential Functions for Protein-Protein Interactions in Aqueous Solutions: Osmotic Second Virial Coefficient Calculations Using the MARTINI Coarse-Grained Force Field. J. Chem. Theory Comput. 2013, 9 (9), 4176-4185.

(26) Javanainen, M.; Martinez-Seara, H.; Vattulainen, I. Excessive Aggregation of Membrane Proteins in the Martini Model. PLoS One 2017, 12 (11), e0187936.

(27) Majumder, A.; Straub, J. E. Addressing the Excessive Aggregation of Membrane Proteins in the MARTINI Model. J Chem. Theory Comput. 2021, 17 (4), 2513-2521.

(28) Schmalhorst, P. S.; Deluweit, F.; Scherrers, R.; Heisenberg, C.P.; Sikora, M. Overcoming the Limitations of the MARTINI Force Field in Simulations of Polysaccharides. J. Chem. Theory Comput. 2017, 13 (10), 5039-5053.

(29) Souza, P. C. T. T.; Alessandri, R.; Barnoud, J.; Thallmair, S.; Faustino, I.; Grünewald, F.; Patmanidis, I.; Abdizadeh, H.; Bruininks, B. M. H. H.; Wassenaar, T. A.; Kroon, P. C.; Melcr, J.; Nieto, V.; Corradi, V.; Khan, H. M.; Domański, J.; Javanainen, M.; Martinez-Seara, H.; Reuter, N.; Best, R. B.; Vattulainen, I.; Monticelli, L.; Periole, X.; Tieleman, D. P.; de Vries, A. H.; Marrink, S. J. Martini 3: A General Purpose Force Field for Coarse-Grained Molecular Dynamics. Nat. Methods 2021, 18 (4), 382-388.

(30) López, C. A.; Sovova, Z.; van Eerden, F. J.; de Vries, A. H.; Marrink, S. J. Martini Force Field Parameters for Glycolipids. J. Chem. Theory Comput. 2013, 9 (3), 1694-1708.

(31) Martini 2 lipid topology for C16:0/18:1 PIP2 (POP2) http://cgmartini.nl/images/parameters/lipids/Phosphatidy linositols/POP2/martini_v2.0_POP2_01.itp (accessed Apr 13, 2021).

(32) Bulacu, M.; Goga, N.; Zhao, W.; Rossi, G.; Monticelli, L.; Periole X.; Tieleman, D. P.; Marrink, S. J. Improved Angle Potentials for Coarse-Grained Molecular Dynamics Simulations. J. Chem. Theory Comput. 2013, 9 (8), 3282-3292.

(33) Borges-Araújo, L.; Fernandes, F. Structure and Lateral Organization of Phosphatidylinositol 4,5-Bisphosphate. Molecules 2020, 25 (17), 3885.

(34) Abraham, M. J.; Murtola, T.; Schulz, R.; Páll, S.; Smith, J. C.; Hess, B.; Lindah, E. Gromacs: High Performance Molecular Simulations through Multi-Level Parallelism from Laptops to Supercomputers. SoftwareX 2015, 1-2, 19-25.

(35) Michaud-Agrawal, N.; Denning, E. J.; Woolf, T. B.; Beckstein, O. MDAnalysis: A Toolkit for the Analysis of Molecular Dynamics 
Simulations. J. Comput. Chem. 2011, 32 (10), 2319-2327.

(36) Pérez, F.; Granger, B. E. IPython: A System for Interactive Scientific Computing.

(37) Harris, C. R.; Millman, K. J.; van der Walt, S. J.; Gommers, R.; Virtanen, P.; Cournapeau, D.; Wieser, E.; Taylor, J.; Berg, S.; Smith, N. J.; Kern, R.; Picus, M.; Hoyer, S.; van Kerkwijk, M. H.; Brett, M.; Haldane, A.; del Río, J. F.; Wiebe, M.; Peterson, P.; Gérard-Marchant, P.; Sheppard, K.; Reddy, T.; Weckesser, W.; Abbasi, H.; Gohlke, C.; Oliphant, T. E. Array Programming with NumPy. Nature 2020, 585 (7825), 357-362.

(38) Virtanen, P.; Gommers, R.; Oliphant, T. E.; Haberland, M.; Reddy, T.; Cournapeau, D.; Burovski, E.; Peterson, P.; Weckesser, W.; Bright, J.; van der Walt, S. J.; Brett, M.; Wilson, J.; Millman, K. J.; Mayorov, N.; Nelson, A. R. J.; Jones, E.; Kern, R.; Larson, E.; Carey, C. J.; Polat, İ.; Feng, Y.; Moore, E. W.; VanderPlas, J.; Laxalde, D.; Perktold, J.; Cimrman, R.; Henriksen, I.; Quintero, E. A.; Harris, C. R.; Archibald, A. M.; Ribeiro, A. H.; Pedregosa, F.; van Mulbregt, P. SciPy 1.0: Fundamental Algorithms for Scientific Computing in Python. Nat. Methods 2020, 17 (3), 261-272.

(39) Fabian, P.; Michel, V.; Grisel, O.; Blondel, M.; Prettenhofer, P.; Weiss, R.; Vanderplas, J.; Cournapeau, D.; Pedregosa, F.; Varoquaux, G.; Gramfort, A.; Thirion, B.; Grisel, O.; Dubourg, V.; Passos, A.; Brucher, M.; Perrot, M.; Duchesnay, É. ScikitLearn: Machine Learning in Python; 2011; Vol. 12.

(40) Rycroft, C. H. VORO++: A Three-Dimensional Voronoi Cell Library in $\mathrm{C}++$. Chaos. American Institute of Physics Inc. December 27, 2009, p 041111.

(41) Hunter, J. D. Matplotlib: A 2D Graphics Environment. Comput Sci. Eng. 2007, 9 (3), 90-95.

(42) Humphrey, W.; Dalke, A.; Schulten, K. VMD: Visual Molecular Dynamics. J. Mol. Graph. 1996, 14 (1), 33-38.

(43) Sousa, F. M.; Lima, L. M. P.; Arnarez, C.; Pereira, M. M.; Melo, M. N. Coarse-Grained Parameterization of Nucleotide Cofactors and Metabolites: Protonation Constants, Partition Coefficients, and Model Topologies. J. Chem. Inf. Model. 2021. Huang, J.; Mackerell, A. D. CHARMM36 All-Atom Additive Protein Force Field: Validation Based on Comparison to NMR Data. J. Comput. Chem. 2013, 34 (25), 2135-2145.

(45) Huang, J.; Rauscher, S.; Nawrocki, G.; Ran, T.; Feig, M.; De Groot, B. L.; Grubmüller, H.; MacKerell, A. D. CHARMM36m: An Improved Force Field for Folded and Intrinsically Disordered Proteins. Nat. Methods 2016, 14 (1), 71-73.

(46) Jo, S.; Kim, T.; Iyer, V. G.; Im, W. CHARMM-GUI: A Web-Based Graphical User Interface for CHARMM. J. Comput. Chem. 2008 29 (11), 1859-1865.

(47) Lee, J.; Cheng, X.; Swails, J. M.; Yeom, M. S.; Eastman, P. K. Lemkul, J. A.; Wei, S.; Buckner, J.; Jeong, J. C.; Qi, Y.; Jo, S.; Pande, V. S.; Case, D. A.; Brooks, C. L.; MacKerell, A. D.; Klauda, J. B.; Im, W. CHARMM-GUI Input Generator for NAMD, GROMACS, AMBER, OpenMM, and CHARMM/OpenMM Simulations Using the CHARMM36 Additive Force Field. J. Chem. Theory Comput. 2016, 12 (1), 405-413.

(48) Wu, E. L.; Cheng, X.; Jo, S.; Rui, H.; Song, K. C.; Dávila-Contreras, E. M.; Qi, Y.; Lee, J.; Monje-Galvan, V.; Venable, R. M.; Klauda, J. B.; Im, W. CHARMM-GUI Membrane Builder toward Realistic Biological Membrane Simulations. Journal of Computational Chemistry. John Wiley and Sons Inc. October 15, 2014, pp 1997-2004.

(49) Jo, S.; Lim, J. B.; Klauda, J. B.; Im, W. CHARMM-GUI Membrane Builder for Mixed Bilayers and Its Application to Yeast Membranes. Biophys. J. 2009, 97 (1), 50-58.

(50) Lee, J.; Patel, D. S.; Ståhle, J.; Park, S. J.; Kern, N. R.; Kim, S.; Lee, J.; Cheng, X.; Valvano, M. A.; Holst, O.; Knirel, Y. A.; Qi, Y.; Jo, S.; Klauda, J. B.; Widmalm, G.; Im, W. CHARMM-GUI Membrane Builder for Complex Biological Membrane Simulations with Glycolipids and Lipoglycans. J. Chem. Theory Comput. 2019, 15 (1), 775-786.

(51) Jo, S.; Kim, T.; Im, W. Automated Builder and Database of Protein/Membrane Complexes for Molecular Dynamics Simulations. PLoS One 2007, 2 (9), e880.

(52) Hess, B.; Bekker, H.; Berendsen, H. J. C.; Fraaije, J. G. E. M. LINCS: A Linear Constraint Solver for Molecular Simulations. J. Comput. Chem. 1997, 18 (12), 1463-1472.
(68) Lupyan, D.; Mezei, M.; Logothetis, D. E.; Osman, R. A Molecular Dynamics Investigation of Lipid Bilayer Perturbation by PIP2. Biophys. J. 2010, 98 (2), 240-247.

(69) Slochower, D. R.; Huwe, P. J.; Radhakrishnan, R.; Janmey, P. A Quantum and All-Atom Molecular Dynamics Simulations of Protonation and Divalent Ion Binding to Phosphatidylinositol 4,5-Bisphosphate (PIP 2 ). J. Phys. Chem. B 2013, 117 (28), 8322-8329.

(70) Slochower, D. R.; Wang, Y.-H.; Radhakrishnan, R.; Janmey, P. A. Physical Chemistry and Membrane Properties of Two Phosphatidylinositol Bisphosphate Isomers. Phys. Chem. Chem. Phys. 2015, 17 (19), 12608-12615.

(71) Bradley, R. P.; Slochower, D. R.; Janmey, P. A.; Radhakrishnan R. Divalent Cations Bind to Phosphoinositides to Induce Ion and Isomer Specific Propensities for Nano-Cluster Initiation in Bilayer Membranes. R. Soc. Open Sci. 2020, 7 (5).

(72) Cheng, T.; Zhao, Y.; Li, X.; Lin, F.; Xu, Y.; Zhang, X.; Li, Y.; Wang,
Darden, T.; York, D.; Pedersen, L. Particle Mesh Ewald: An
$\mathrm{N} \cdot \log (\mathrm{N})$ Method for Ewald Sums in Large Systems. J. Chem Phys. 1993, 98 (12), 10089-10092.

Chem. Phys. 1985, 83 (8), 4069-4074.

Crystals: A New Molecular Dynamics Method. J. Appl. Phys. Weissig, H.; Shindyalov, I. N.; Bourne, P. E. The Protein Data ( GitHub - marrink-lab/vermouth-martinize: Describe and Herzog, F. A.; Braun, L.; Schoen, I.; Vogel, V. Improved Side Martini Straight: Boosting Performance Using a Shorter Bussi, G.; Donadio, D.; Parrinello, M. Canonical Sampling 014101.

Kanduč, M.; Kulig, W.; Lamberg, A.; Loison, C.; Lyubartsev, A Retegan, M.; Róg, T.; Santuz, H.; Tynkkynen, J. Toward Atomistic Resolution Structure of Phosphatidylcholine . . Phys. Chem. B 2015, 119 (49), 15075-15088. Phys. Lipids 2014, 182 (January), 38-51.

All-Atom Force Field for Acyclic Polyalcohols, Acyclic Bank. Nucleic Acids Research. Oxford University Press January 
R.; Lai, L. Computation of Octanol-Water Partition Coefficients by Guiding an Additive Model with Knowledge. J. Chem. Inf. Model. 2007, 47 (6), 2140-2148.

(73) Tetko, I. V.; Tanchuk, V. Y.; Kasheva, T. N.; Villa, A. E. P. Estimation of Aqueous Solubility of Chemical Compounds Using E-State Indices. J. Chem. Inf. Comput. Sci. 2001, 41 (6), 1488-1493.

(74) Tetko, I. V.; Bruneau, P. Application of ALOGPS to Predict 1Octanol/Water Distribution Coefficients, LogP, and LogD, of AstraZeneca in-House Database. J. Pharm. Sci. 2004, 93 (12), 3103-3110.

(75) Tetko, I. V.; Poda, G. I. Application of ALOGPS 2.1 to Predict Log D Distribution Coefficient for Pfizer Proprietary Compounds. J. Med. Chem. 2004, 47 (23), 5601-5604.

(76) Klopman, G.; Li, J. Y.; Wang, S.; Dimayuga, M.; Wang, S.; Dimayuga, M. Computer Automated Log P Calculations Based on an Extended Group Contribution Approach. J. Chem. Inf. Comput. Sci. 1994, 34 (4), 752-781.

(77) ChemAxon Chemicalize http://www.chemicalize.org/.

(78) Lay, W. K.; Miller, M. S.; Elcock, A. H. Optimizing Solute-Solute Interactions in the GLYCAM06 and CHARMM36 Carbohydrate Force Fields Using Osmotic Pressure Measurements. J. Chem. Theory Comput. 2016, 12 (4), 1401-1407.

(79) Davis, D. J.; Burlak, C.; Money, N. P. Osmotic Pressure of Fungal Compatible Osmolytes. Mycol. Res. 2000, 104 (7), 800-804.

(80) Brown, W. H. Organic Chemistry:; Brooks/Cole Cengage Learning, 2009.

(81) van Paridon, P. A.; de Kruijff, B.; Ouwerkerk, R.; Wirtz, K. W. A. Polyphosphoinositides Undergo Charge Neutralization in the Physiological PH Range: A 31P-NMR Study. Biochim. Biophys. Acta - Lipids Lipid Metab. 1986, 877 (1), 216-219.

(82) Kooijman, E. E. E.; King, K. E. E.; Gangoda, M.; Gericke, A. Ionization Properties of Phosphatidylinositol Polyphosphates in Mixed Model Membranes. Biochemistry 2009, 48 (40), 9360-9371.

(83) Graber, Z. T.; Thomas, J.; Johnson, E.; Gericke, A.; Kooijman, E. E. Effect of H-Bond Donor Lipids on Phosphatidylinositol3,4,5-Trisphosphate Ionization and Clustering. Biophys. J. 2018, 114 (1), 126-136.

(84) Bradshaw, J. P.; Bushby, R. J.; Giles, C. C. .; Saunders, M. R.; Saxena, A. The Headgroup Orientation of Dimyristoylphosphatidylinositol-4-Phosphate in Mixed Lipid Bilayers: A Neutron Diffraction Study. Biochim. Biophys. Acta - Biomembr. 1997, 1329 (1), 124-138.

(85) Zhou, C.; Garigapati, V.; Roberts, M. F. Short-Chain Phosphatidylinositol Conformation and Its Relevance to Phosphatidylinositol-Specific Phospholipase C. Biochemistry 1997, 36 (50), 15925-15931.

(86) Bradshaw, J. P.; Bushby, R. J.; Giles, C. C.; Saunders, M. R Orientation of the Headgroup of Phosphatidylinositol in a Model Biomembrane as Determined by Neutron Diffraction. Biochemistry 1999, 38 (26), 8393-8401.

(87) Kishore, A. I.; Prestegard, J. H. Molecular Orientation and Conformation of Phosphatidylinositides in Membrane Mimetics Using Variable Angle Sample Spinning (VASS) NMR. Biophys. J. 2003, 85 (6), 3848-3857.

(88) Fernandes, F.; Loura, L. M. S.; Fedorov, A.; Prieto, M. Absence of Clustering of Phosphatidylinositol- $(4,5)$-Bisphosphate in
Fluid Phosphatidylcholine. J. Lipid Res. 2006, 47 (7), 15211525.

(89) Gericke, A. Is Calcium Fine-Tuning PhosphoinositideMediated Signaling Events Through Clustering? Biophysical Journal. Biophysical Society June 5, 2018, pp 2483-2484.

(90) Ellenbroek, W. G.; Wang, Y. H.; Christian, D. A.; Discher, D. E.; Janmey, P. A.; Liu, A. J. Divalent Cation-Dependent Formation of Electrostatic PIP2 Clusters in Lipid Monolayers. Biophys. J. 2011, 101 (9), 2178-2184.

(91) van den Bogaart, G.; Meyenberg, K.; Risselada, H. J.; Amin, H.; Willig, K. I.; Hubrich, B. E.; Dier, M.; Hell, S. W.; Grubmüller, H.; Diederichsen, U.; Jahn, R. Membrane Protein Sequestering by Ionic Protein-Lipid Interactions. Nature 2011, 479 (7374), 552-555.

(92) Wang, J.; Richards, D. A. Segregation of PIP2 and PIP3 into Distinct Nanoscale Regions within the Plasma Membrane. Biol. Open 2012, 1 (9), 857-862.

(93) Golebiewska, U.; Nyako, M.; Woturski, W.; Zaitseva, I.; McLaughlin, S. Diffusion Coefficient of Fluorescent Phosphatidylinositol 4,5-Bisphosphate in the Plasma Membrane of Cells. Mol. Biol. Cell 2008, 19 (4), 1663-1669.

(94) Han, K.; Gericke, A.; Pastor, R. W. Characterization of Specific Ion Effects on PI(4,5)P 2 Clustering: Molecular Dynamics Simulations and Graph-Theoretic Analysis. J. Phys. Chem. B 2020, 124 (7), 1183-1196.

(95) Borges-Araújo, L.; Domingues, M. M.; Fedorov, A.; Santos, N. C.; Melo, M. N.; Fernandes, F. Acyl-Chain Saturation Regulates the Order of Phosphatidylinositol 4,5-Bisphosphate Nanodomains [Poster Session]. 7th European Joint Theoretical/Experimental Meeting on Membranes - Book of Abstracts. Graz, Austria 2021, pp PP02-1-B.

(96) Lemmon, M. A.; Ferguson, K. M. Signal-Dependent Membrane Targeting by Pleckstrin Homology (PH) Domains. Biochem. J. 2000, 350 (1), 1-18.

(97) Garcia, P.; Gupta, R.; Shah, S.; Morris, A. J.; Rudge, S. A. Scarlata, S.; Petrova, V.; McLaughlin, S.; Rebecchi, M. J. The Pleckstrin Homology Domain of Phospholipase C-Delta 1 Binds with High Affinity to Phosphatidylinositol 4,5Bisphosphate in Bilayer Membranes. Biochemistry 1995, 34 (49), 16228-16234

(98) Lemmon, M. A. Pleckstrin Homology (PH) Domains and Phosphoinositides. Biochem. Soc. Symp. 2007, 74 (1), 81.

(99) Khan, H. M.; Souza, P. C. T.; Thallmair, S.; Barnoud, J.; De Vries A. H.; Marrink, S. J.; Reuter, N. Capturing Choline-Aromatics Cation-חInteractions in the MARTINI Force Field. J. Chem Theory Comput. 2020, 16 (4), 2550-2560.

(100) D’Avanzo, N.; Cheng, W. W. L.; Doyle, D. A.; Nichols, C. G. Direct and Specific Activation of Human Inward Rectifier K+ Channels by Membrane Phosphatidylinositol 4,5Bisphosphate. J. Biol. Chem. 2010, 285 (48), 37129-37132.

(101) Lacin, E.; Aryal, P.; Glaaser, I. W.; Bodhinathan, K.; Tsai, E.; Marsh, N.; Tucker, S. J.; Sansom, M. S. P.; Slesinger, P. A. Dynamic Role of the Tether Helix in PIP2-Dependent Gating of a G Protein-Gated Potassium Channel. J. Gen. Physiol. 2017, 149 (8), 799-811. 
Authors are required to submit a graphic entry for the Table of Contents (TOC) that, in conjunction with the manuscript title, should give the reader a representative idea of one of the following: A key structure, reaction, equation, concept, or theorem, etc., that is discussed in the manuscript. Consult the journal's Instructions for Authors for TOC graphic specifications.

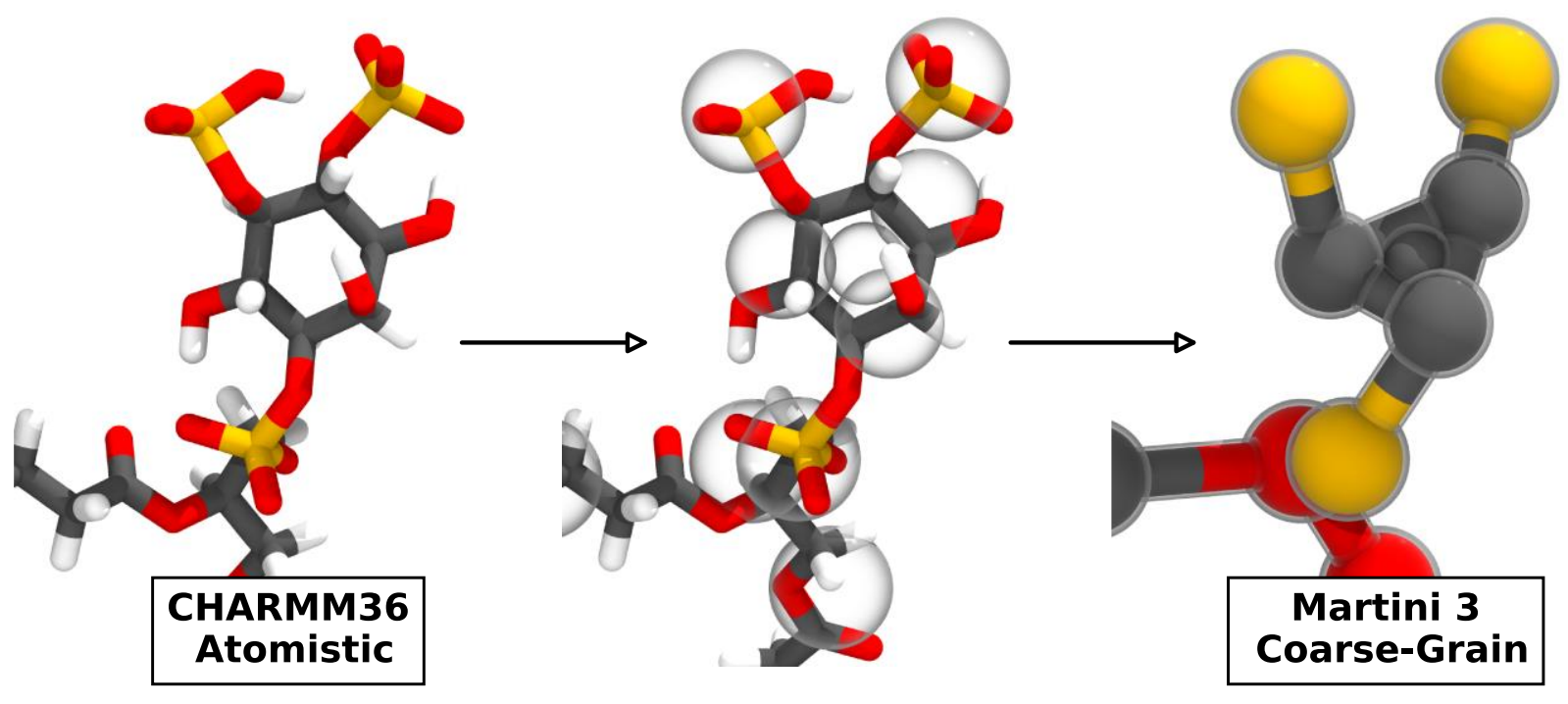

